\title{
Safety and Efficacy of Anticoagulation in Patients with Cirrhosis: A Meta-Analysis
}

\author{
Huan Chen, Jiaming Lei, Sicheng Liang, Gang Luo, Mingming Deng $(\mathbb{D}$, and Muhan Lü \\ Department of Gastroenterology, The Affiliated Hospital of Southwest Medical University, Luzhou, Sichuan Province, China \\ Correspondence should be addressed to Mingming Deng; dengmingming@swmu.edu.cn and Muhan Lü; lvmuhan@swmu.edu.cn
}

Received 25 August 2020; Revised 6 January 2021; Accepted 8 February 2021; Published 29 April 2021

Academic Editor: Quirino Lai

Copyright (C) 2021 Huan Chen et al. This is an open access article distributed under the Creative Commons Attribution License, which permits unrestricted use, distribution, and reproduction in any medium, provided the original work is properly cited.

\begin{abstract}
Background and Aims. Portal vein thrombosis is a serious adverse event that occurs during liver cirrhosis. We performed a metaanalysis to evaluate the safety and efficacy of anticoagulant therapy and prophylactic anticoagulant therapy in cirrhosis patients with (/without) portal vein thrombosis. Methods. Eligible comparative studies were identified by searching the following electronic databases: PubMed, Embase, Cochrane Library, Web of Science, and CNKI. A meta-analysis was performed to calculate odds ratios and 95\% confidence intervals using fixed-effects models. Recanalization and thrombus progression were defined as the primary outcomes. Secondary outcomes included adverse events and death mortality. Results. A total of 3479 patients were included in this analysis. Compared with the control group, the recanalization rate in the anticoagulant therapy group was increased $(P<0.00001)$ in patients with cirrhosis and portal vein thrombosis without increasing adverse events. Multiple use of enoxaparin in small doses is safer than single large doses $(P=0.004)$. Direct oral anticoagulants are more effective $(P<0.00001)$ and safer than traditional anticoagulants. Prophylactic anticoagulant therapy can effectively prevent portal vein thrombosis formation $(P<0.00001)$. Conclusions. Anticoagulation therapy can treat or prevent portal vein thrombosis in patients with liver cirrhosis and is a relatively safe treatment.
\end{abstract}

\section{Introduction}

Portal vein thrombosis (PVT) is a common adverse event of liver cirrhosis, and its incidence increases as liver disease progresses and is even higher in patients with various portal hypertension procedures [1-3]. Patients with acute and severe PVT may experience symptoms, such as fever, abdominal pain, ascites, and splenomegaly, but many patients do not exhibit symptoms in the early stage of onset [4]. The hidden onset of PVT can cause significant harms to patients, including intestinal congestion and necrosis, secondary serious infections, increased risk of bleeding from esophageal varices rupture of the stomach, increased decompensation of the liver, more intraoperative and postoperative adverse events, and higher mortality $[5,6]$. Therefore, to improve patient prognosis, timely and effective treatments of portal PVT are very important. As one of the main treatments of PVT, anticoagulation has received increasing attention in recent years, and prophylactic anticoagulation has even been proposed for patients at high risk of PVT. However, no definitive conclusion on anticoagulation effectiveness and safety has been reported. Some studies found that the recanalization rate of PVT after anticoagulation treatment is greater than $80 \%[7,8]$. However, other studies showed that anticoagulation treatment might be ineffective for PVT $[9,10]$. Therefore, it is necessary to analyze relevant previous studies. This article is divided into two parts, namely, anticoagulation and prophylactic anticoagulation therapy, and both topics are analyzed using and metaanalysis to provide a reference for clinicians to treat or prevent PVT in patients with cirrhosis.

\section{Materials and Methods}

2.1. Document Retrieval. "Cirrhosis," "liver cirrhosis," "liver cirrhoses," "hepatic cirrhosis," "portal vein," "thrombosis," "thromboses," "thrombus," "blood clot," "anticoagulant," "anticoagulation," "anticoagulant therapy," "thrombin 
inhibitors" and other keywords were used to search databases, including PubMed, Embase, Cochrane Library, Web of Science, Wanfang, CNKI, and Weipu Database. The studies reported randomized controlled trials (RCT) and nonrandomized controlled trials (nRCT). No language limitations were imposed. This study included papers published up to December 2019.

2.2. Inclusion Criteria. (1) RCT or nRCT; (2) study subjects were patients older than 18 years of age with liver cirrhosis at any stage attributed to various etiologies, and there were no restrictions on the race, nationality, or region; (3) the observation group was administered anticoagulants for anticoagulation, and the control group was treated with placebo or blank control, different anticoagulants, or different doses or treatment times with the same anticoagulant; (4) data reported should include these outcome indicators: portal vein recanalization or new onset, bleeding events, death, and other adverse events, including the new onset of decompensation of liver function, ascites, spontaneous peritonitis, sepsis, hepatorenal syndrome, or hepatic encephalopathy.

2.3. Exclusion Criteria. (1) Nonclinical research; (2) studies for which a full text is not available; (3) republished literatures; (4) studies that do not provide complete data; (5) research subjects are noncirrhotic patients; (6) subjects have an underlying primary blood disease, membranous obstruction of the inferior vena cava, or preexisting extrahepatic thrombosis; (7) interventions other than anticoagulation; (8) research that is not germane to our subject.

2.4. Screening and Quality Evaluation. After reading the titles and abstracts of all the retrieved studies, preliminary screening was performed. The full text of the documents that passed the preliminary screening was read to exclude documents that clearly do not meet the requirements or are duplicate studies. The Cochrane bias risk assessment tool was used to assess the bias risk of included RCTs, and the Newcastle-Ottawa Scale (NOS) was used to assess the quality of included nRCTs.

2.5. Data Extraction and Statistical Analysis. Data extracted from each study included the following: first author, year of publication, country of publication, number of patients, liver function score, specific interventions, overall follow-up time, portal vein recanalization or new occurrence, bleeding events, other adverse events, and death.

\section{Results}

A total of 403 articles passed the preliminary screening, and 302 were excluded due to noncompliance of the study subjects or the use of intervention methods other than anticoagulation. In addition, 29 were nonclinical studies, and the full text of 16 articles could not be obtained. Moreover, 20 articles did not meet the requirements. Thus, thirty-six papers $[2,9,11-44]$ were ultimately selected to complete this meta-analysis (Figure 1).

3.1. Basic Characteristics of Included Literatures. Of the 36 selected papers, 21 were reported in English, and 15 were in Chinese. Of the selected papers, 11 studies reported RCTs, and 25 reported nRCTs. The study sites included China, the United States, Europe, Japan, and other places. The dates of publication ranged from 2005 to 2019 , and a total of 3479 patients were included. The basic characteristics of the included studies are provided in Table 1.

3.2. Bias Risk Assessment. Cochrane bias risk assessment tool and NOS scale were selected for evaluation, as shown in Figures 2(a) and 2(b) and Table 2.

\subsection{Statistical Results of Anticoagulant Therapy.} Figure 3(a) shows that the PVT recanalization rate in the observation group (anticoagulation) is increased compared with the control group, and the results are statistically significant $(\mathrm{OR}=5.10,95 \% \mathrm{CI}: 3.93 \sim 6.61, P<0.00001)$. Subgroup analysis based on different drugs (other represents other anticoagulants, heparin, and/or warfarin combined with others) (Figure 3(b)) more specifically shows that different anticoagulants have therapeutic effects on PVT. Figure $3 \mathrm{C}$ shows that the thrombus progression or new thrombus formation in the observation group was reduced compared with the control group $(\mathrm{OR}=0.22,95 \% \mathrm{CI}$ : $0.13 \sim 0.37, P<0.00001)$. Compared with the control group, anticoagulation did not increase the incidence of bleeding events $(\mathrm{OR}=0.70,95 \% \mathrm{CI}: 0.49 \sim 1.02, P=0.06)$ or the incidence of other adverse events $(\mathrm{OR}=0.62,95 \% \mathrm{CI}$ : $0.37 \sim 1.02, P=0.06)$, but the mortality rate was reduced $(\mathrm{OR}=0.25,95 \%$ CI: $0.08 \sim 0.81, P=0.02$ ) (Figures $2(\mathrm{~d})$ 2(f)) (see Supplementary Figure 1 for histogram).

\subsubsection{Effect of Anticoagulant Therapy with Different Enox-} aparin Doses. When different doses of enoxaparin were used for anticoagulation, the same effects were noted in the observation group $(1.0 \mathrm{mg} / \mathrm{kg} \mathrm{q} 12 \mathrm{~h})$ and the control group $(1.5 \mathrm{mg} / \mathrm{kg} \mathrm{q} 24 \mathrm{~h})$ of patients with liver cirrhosis and PVT $(\mathrm{OR}=1.03$, 95\% CI: $0.47 \sim 2.27, P=0.94)$ (Figure 4(a)), but the incidence of bleeding events was reduced in the former $(\mathrm{OR}=0.24,95 \% \mathrm{CI}: 0.09 \sim 0.62, P=0.004)$ (Figure 4(b)). No significant difference in the incidence of other adverse events was between the two groups (OR $=1.43,95 \%$ CI: $0.67 \sim 3.08$, $P=0.36$ ) (Figure 4(c)) (see Supplementary Figure 2 for histogram).

3.3.2. Therapeutic Effects of Direct Oral Anticoagulants (DOAC) vs. Traditional Anticoagulants. The thrombus recanalization rate in the observation group (DOAC) was increased compared with the control group (traditional anticoagulant $) \quad(\mathrm{OR}=33.04, \quad 95 \% \quad \mathrm{CI}: \quad 9.23 \sim 118.28$, $P<0.00001)$ (Figure 5(a)). Apparently, bleeding $(\mathrm{OR}=0.35$, 95\% CI: $0.15 \sim 0.81, P=0.01$ ) and other adverse events 


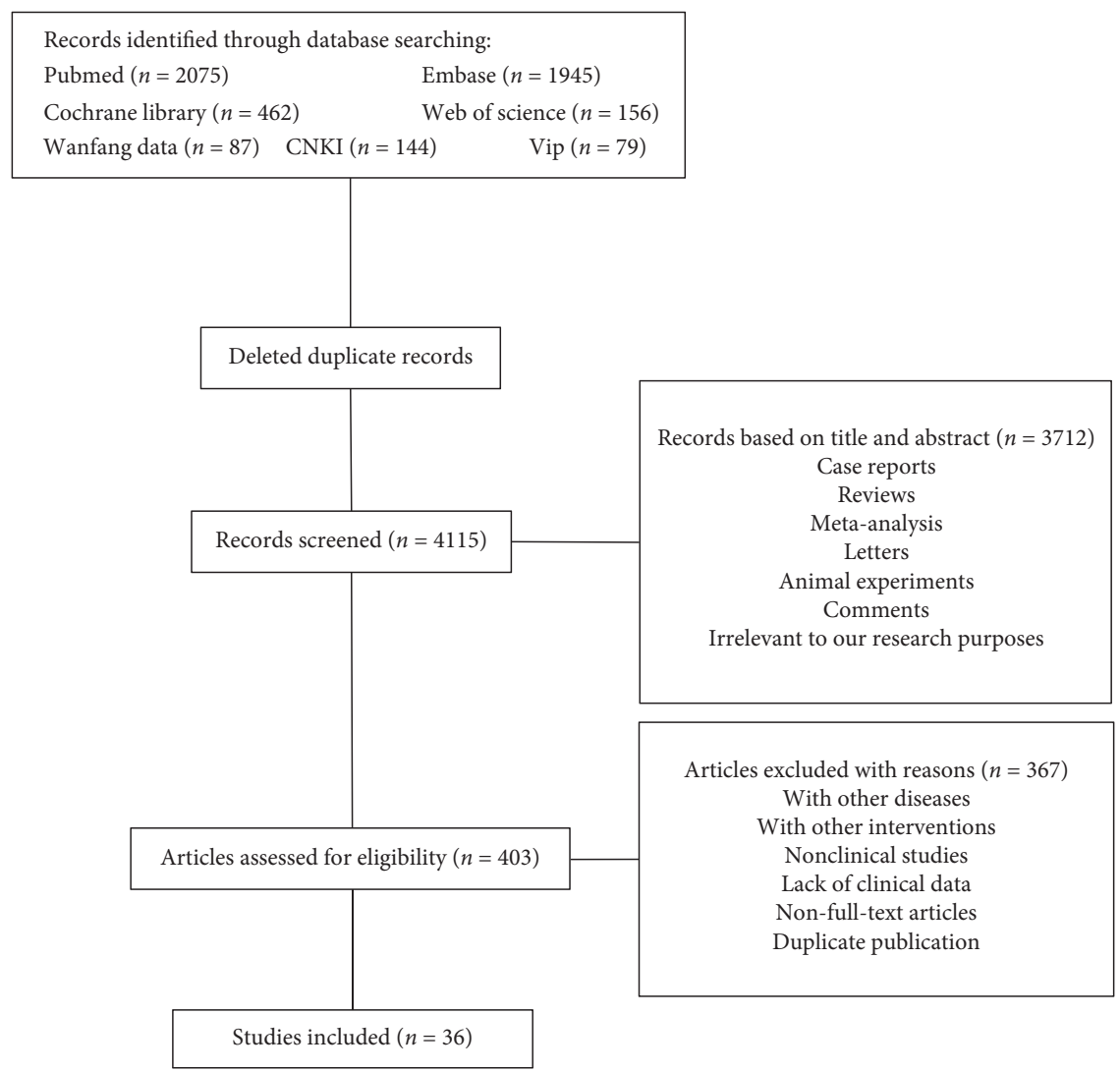

Figure 1: Flow chart of literature screening.

$(\mathrm{OR}=0.16,95 \% \mathrm{CI}: 0.05 \sim 0.49, P=0.001)$ in the observation group were reduced compared with the traditional anticoagulant group (Figures 5(b) and 5(d)). However, given the significant heterogeneity, the random effect model was used to merge the data. And the differences between the two groups were not statistically significant, including the incidence of bleeding events $(\mathrm{OR}=0.51,95 \% \mathrm{CI}$ : $0.03 \sim 9.83$, $P=0.65)$, risk of other adverse events $(\mathrm{OR}=0.19,95 \% \mathrm{CI}$ : $0.00 \sim 35.04, P=0.53)$, and death $(\mathrm{OR}=0.37,95 \% \mathrm{CI}$ : 0.01 22.19, $P=0.64$ ) (Figures 5(c), 5(e) and 5(f)) (see Supplementary Figure 3 for histogram).

\subsection{Statistical Results of Prophylactic Anticoagulation}

3.4.1. Effect and Safety of Prophylactic Anticoagulation. The rate of PVT in the observation group (prophylactic anticoagulation treatment) was reduced compared with the control group, and the results were statistically significant $(\mathrm{OR}=0.23,95 \% \mathrm{CI}: 0.14 \sim 0.37, P<0.00001)$ (Figure 6(a)). Using subgroup analysis, we found that the incidence of thrombosis in patients after splenectomy was significantly reduced compared with the control group ( $\mathrm{OR}=0.17,95 \%$ CI: $0.06 \sim 0.48, P=0.0008$ ), but the difference was not significant in patients with liver cirrhosis after cancer resection (OR $=0.22,95 \% \mathrm{CI}: 0.03 \sim 1.65, P=0.14$ ) or no operation (OR $=0.25,95 \% \mathrm{CI}: 0.06 \sim 1.01, P=0.05$ ) (Figure 6(b)). The incidence of bleeding events in the observation group was increased compared with the control group (OR $=3.33,95 \%$
CI: 1.07 10.37, $P=0.04$ ) (Figure 6(c)) (see Supplementary Figure 4 for histogram).

3.4.2. The Effect of Prophylactic Anticoagulation with Different Drugs. During preventive anticoagulation, the rate of thrombosis formation did not differ in the observation group (warfarin) and the control group (aspirin) $(\mathrm{OR}=0.33$, 95\% CI: 0.03 3.76, $P=0.37$ ) (Figure 7) (see Supplementary Figure 5 for histogram).

3.4.3. Integration of Traditional Chinese and Western Medicine to Prevent PVT Formation. In anticoagulation therapies, the addition of drugs to promote blood circulation and prevent blood stasis can reduce the incidence of portal vein thrombosis $(\mathrm{OR}=0.24,95 \% \mathrm{CI}: 0.17 \sim 0.34, P<0.00001)$ (Figure 8(a)). No significant differences in PLT (MD $=-58.71,95 \% \mathrm{CI}:-203.41 \sim 86.00, P=0.43)$, APTT $(\mathrm{MD}=-2.06,95 \% \mathrm{CI}:-5.22 \sim 1.10, \quad P=0.20)$, or $\mathrm{PT}$ (MD $=-0.65,95 \% \mathrm{CI}:-2.05 \sim 0.75, P=0.36)$ were noted between the two groups (Figures $8(\mathrm{~b})-8(\mathrm{~d})$ ) (see Supplementary Figure 6 for histogram).

\section{Discussion}

The liver is an important organ that maintains the balance of the hemostatic system. As cirrhosis progresses, disorders of the coagulation and fibrinolytic system may occur, which can easily lead to bleeding and thromboembolism in 


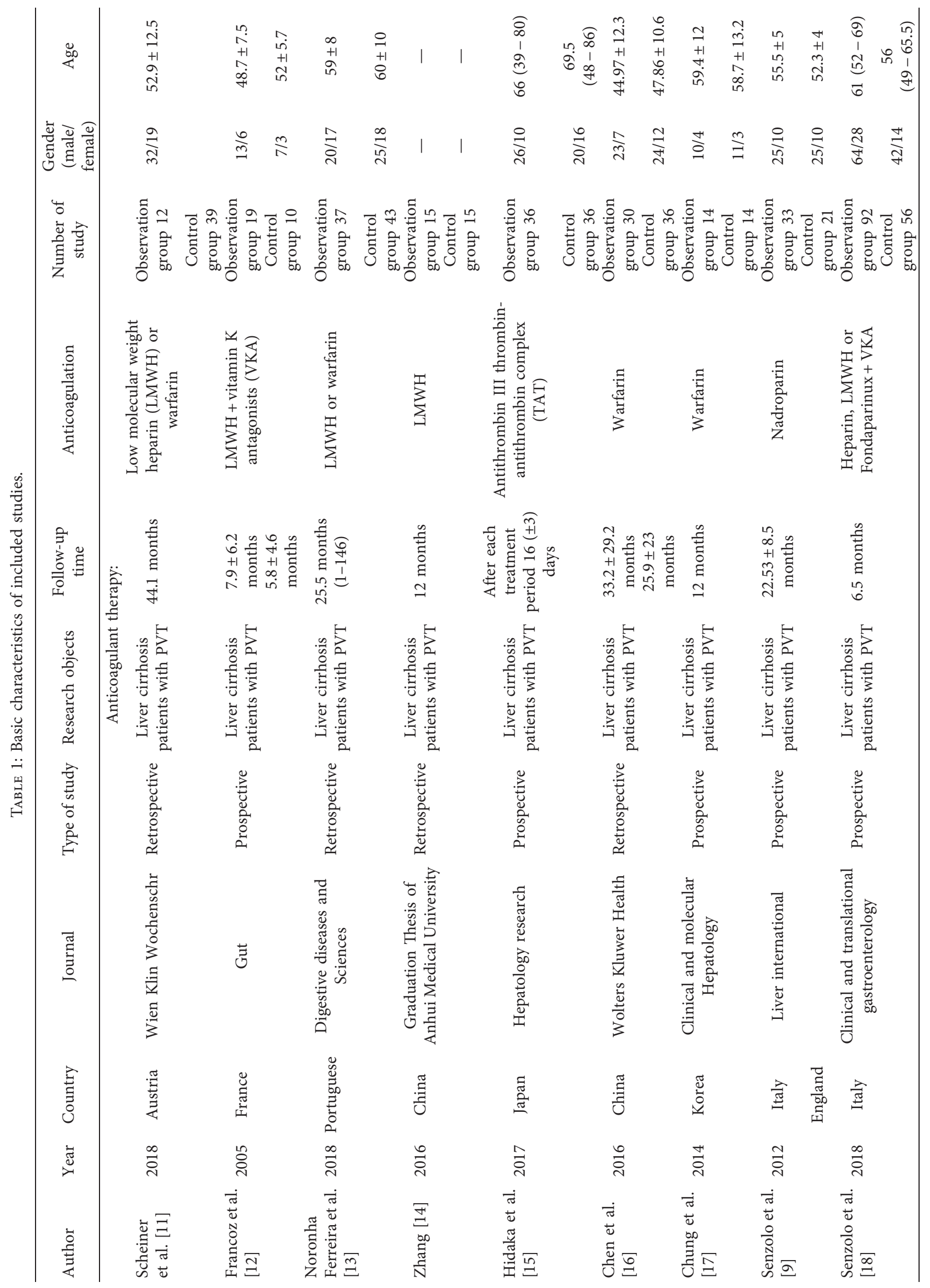




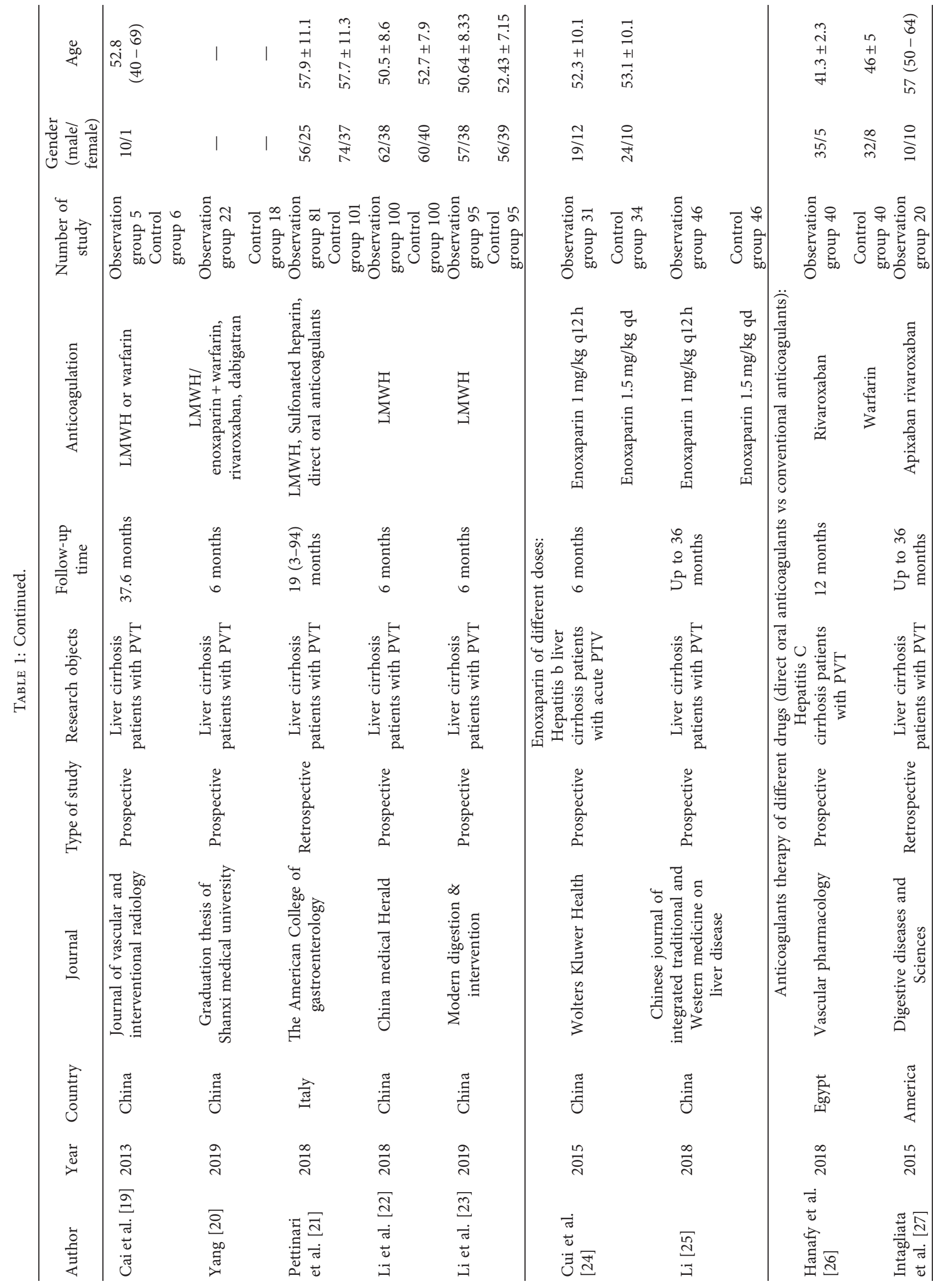




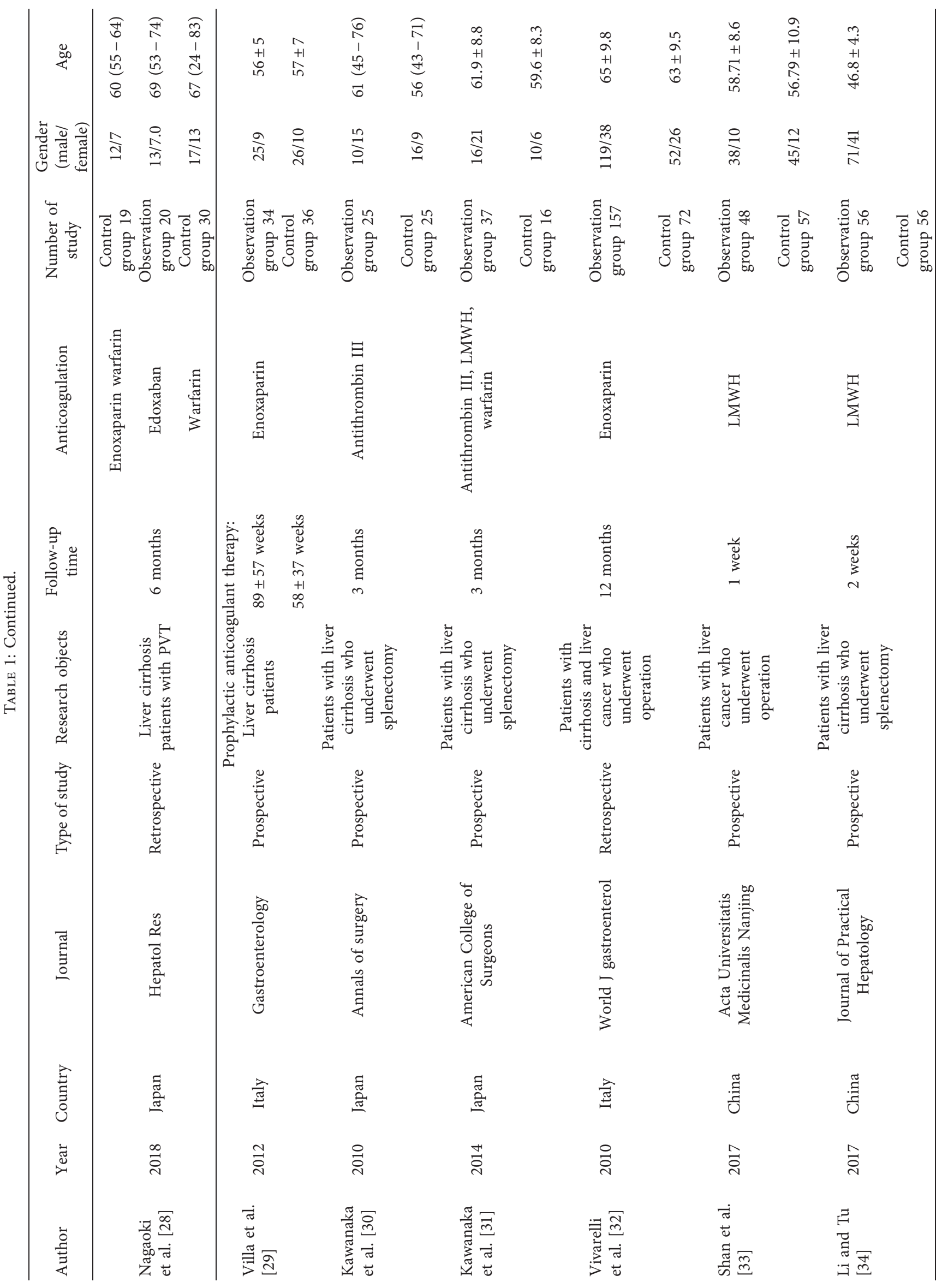




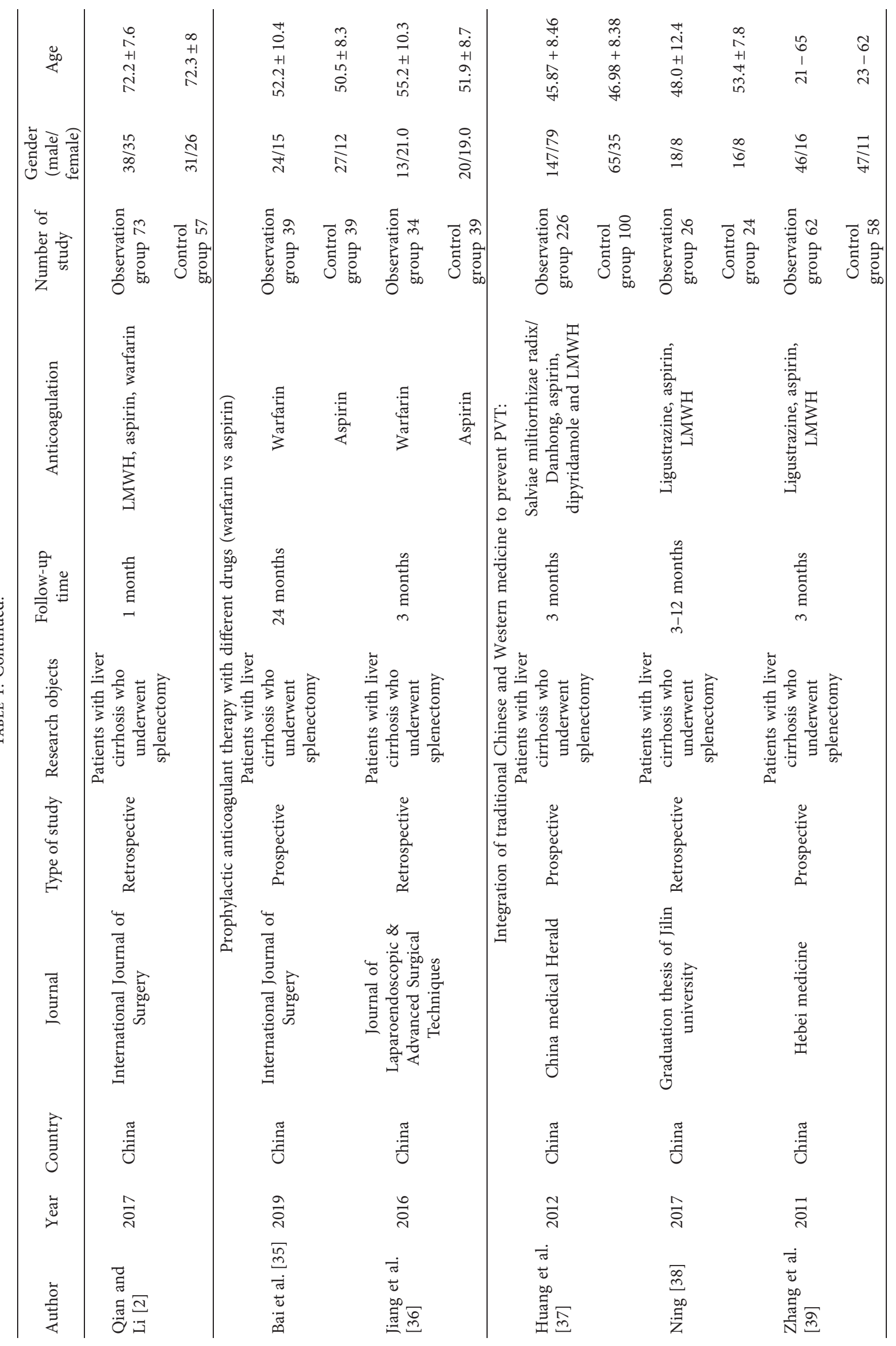




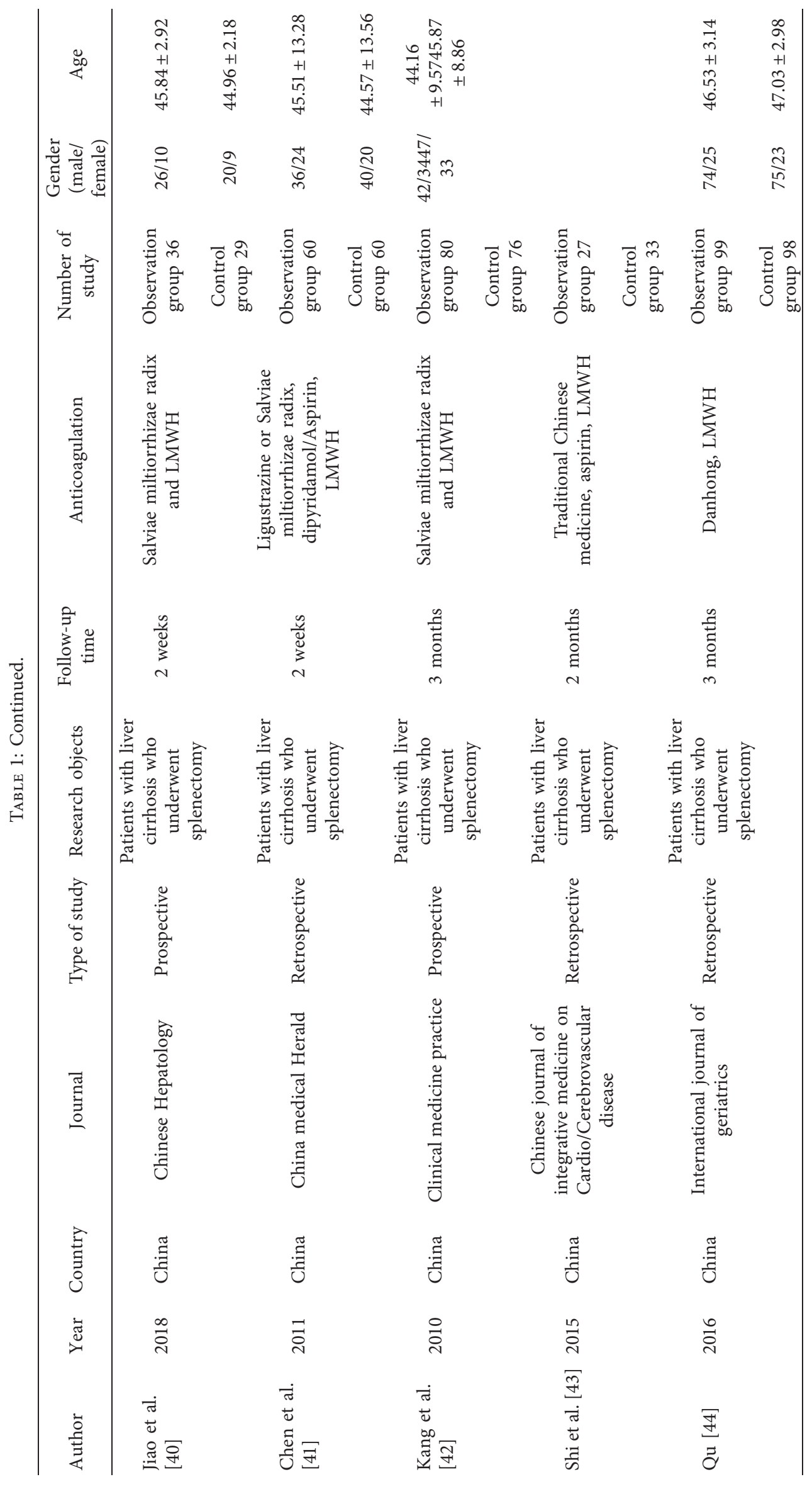




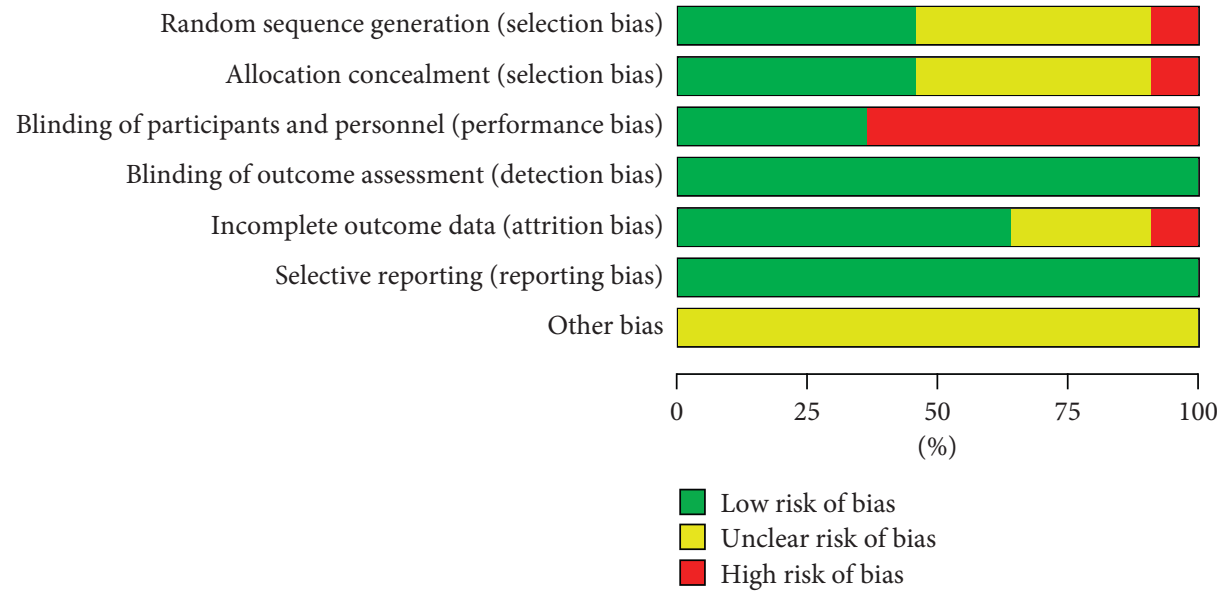

(a)
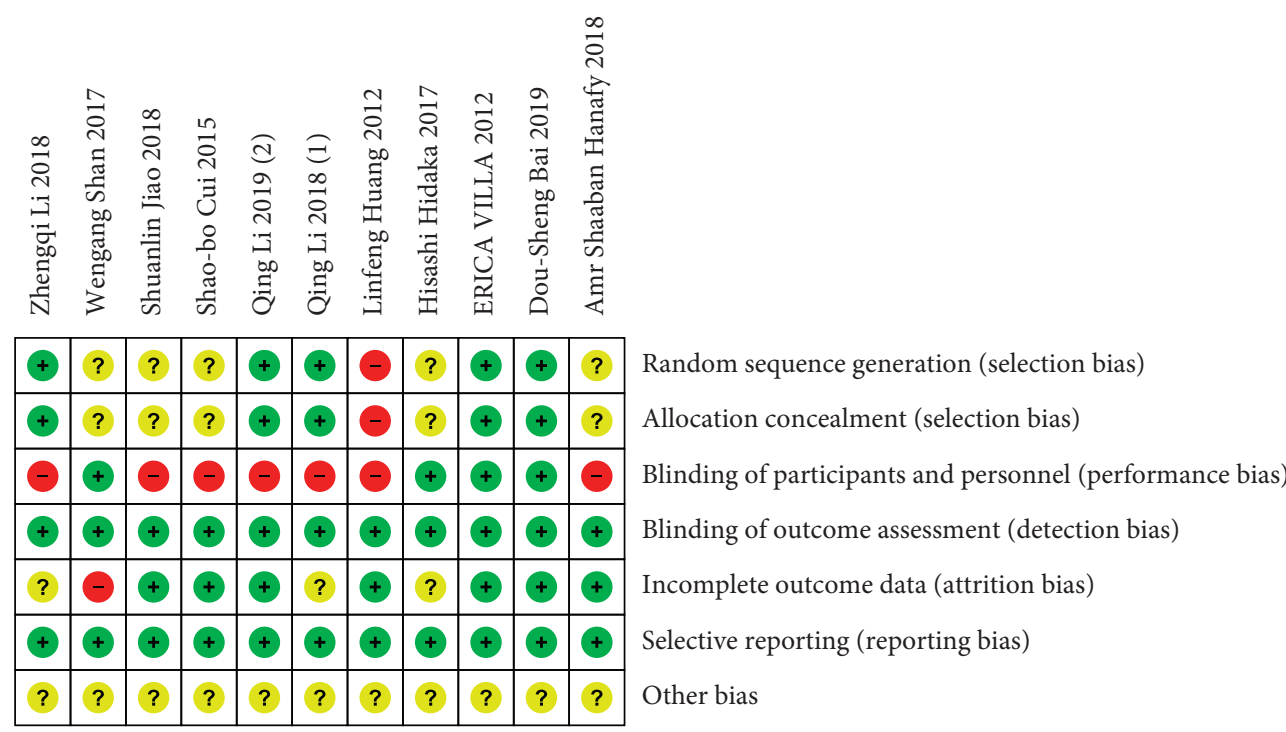

(b)

Figure 2: Bias analysis: (a) A review of the authors' judgments about each risk of bias item presented as percentages; (b) A review of the authors' judgments about each risk of bias item for included studies.

patients. PVT, a serious adverse event of liver cirrhosis, is closely related to the hemodynamics of advanced portal hypertension. Its treatment methods include anticoagulation, thrombolysis, transjugular intrahepatic portal vein shunt (TIPS), and surgery. At present, few studies have assessed thrombolysis, and interventional therapy is generally suitable for patients with acute and severe PVT. Surgery is mainly used for patients with severe adverse events, such as uncontrollable gastrointestinal bleeding and intestinal necrosis caused by thrombosis. As a relatively noninvasive and simple treatment, anticoagulation represents one of the main clinical treatments for PVT. Anticoagulation therapy has achieved excellent results in the treatment of many cirrhosis patients with PVT and even patients with portal vein cavernous tumors [45-48]. However, the use of anticoagulants may cause some side effects, such as elevated liver enzymes, thrombocytopenia, prolonged prothrombin time, and even life-threatening cases
[21, 49]. Therefore, the effectiveness and safety of anticoagulation therapy were further discussed in this article.

In terms of therapeutic anticoagulation, the results showed that anticoagulation prevents thrombus progression and increases the thrombosis recanalization rate. It is worth mentioning that in a study [50], the portal cavernomas were disappeared in two patients after anticoagulation. In addition, compared with the control group, bleeding events and other adverse events did not increase, and the mortality rate was decreased in the observation group. These results show that anticoagulation can treat cirrhosis PVT and improve patient survival without increasing side effects. Studies indicated that microthrombosis in the liver sinus exists in patients with cirrhosis [51, 52]. Microthrombi can increase portal pressure and cause intimal fibrosis and venous occlusion, eventually causing adjacent liver cells to be lost and replaced by fibrous tissue. Anticoagulation can improve liver fibrosis by combating microthrombosis, further improving 
TABle 2: The quality of studies with NOS scores.

\begin{tabular}{|c|c|c|c|c|}
\hline Studies & Selection & Comparability & Outcome & Stars \\
\hline Scheiner et al. 2018 [11] & 4 & 1 & 2 & 7 \\
\hline Francoz et al. $2005[12]$ & 4 & 2 & 3 & 9 \\
\hline Noronha Ferreira et al. 2018 [13] & 4 & 2 & 3 & 9 \\
\hline Zhang $2016[14]$ & 4 & 0 & 3 & 7 \\
\hline Chen et al. 2016 [16] & 4 & 2 & 3 & 9 \\
\hline Chung et al. 2014 [17] & 4 & 2 & 3 & 9 \\
\hline Senzolo et al. 2012 [9] & 3 & 2 & 2 & 7 \\
\hline Senzolo et al. 2018 [18] & 4 & 2 & 2 & 8 \\
\hline Cai et al. 2013 [19] & 3 & 0 & 3 & 6 \\
\hline Yang 2019 [20] & 4 & 0 & 3 & 7 \\
\hline Pettinari et al. 2018 [21] & 4 & 1 & 3 & 8 \\
\hline Intagliata et al. 2016 [27] & 4 & 2 & 3 & 9 \\
\hline Nagaoki et al. 2018 [28] & 4 & 2 & 3 & 9 \\
\hline Kawanaka et al. 2010 [30] & 3 & 3 & 3 & 8 \\
\hline Kawanaka et al. 2014 [31] & 4 & 1 & 3 & 8 \\
\hline Vivarelli et al. 2010 [32] & 4 & 1 & 3 & 8 \\
\hline $\mathrm{Li}$ and $\mathrm{Tu} 2017$ [34] & 4 & 1 & 2 & 7 \\
\hline Harding et al. [6] & 4 & 1 & 3 & 8 \\
\hline Jiang et al. 2016 [36] & 4 & 2 & 3 & 9 \\
\hline Ning 2017 [38] & 4 & 2 & 3 & 9 \\
\hline Zhang et al. 2011 [39] & 4 & 0 & 3 & 7 \\
\hline Chen et al. 2011 [41] & 4 & 0 & 3 & 7 \\
\hline Kang and Zhang 2010 [42] & 4 & 1 & 3 & 8 \\
\hline Shi et al. 2015 [43] & 4 & 1 & 3 & 8 \\
\hline Qu 2016 [44] & 4 & 2 & 3 & 9 \\
\hline
\end{tabular}

liver function and reducing portal hypertension. Francoz et al. [12] found that liver function and renal function were improved in patients treated with enoxaparin. He also noted that enoxaparin could reduce intestinal cell damage by improving intestinal microcirculation, thereby reducing bacterial translocation. The Thrombosis Canada and 7th International Coagulation in Liver Disease Conference recommended liver transplant candidates with PVT for anticoagulation therapy and pointed out nontransplant candidates with acute PVT may also benefit [53]. Therefore, anticoagulation represents a safe, effective, and reliable option for patients with cirrhosis PVT, even those with poor liver function.

The 2016 Consensus of the Italian Society of Hepatology and the Italian Medical Association: Hemostasis Balance of Cirrhosis reported that thromboprophylaxis is not absolutely contraindicated in patients with cirrhosis [54]. However, through repeated searches of these literature libraries, only one controlled study [29] on preventive anticoagulation in nonsurgical cirrhosis patients was identified. Villa et al. found that enoxaparin was safe in preventing PVT in cirrhosis patients and delayed the occurrence of hepatic decompensation. However, related studies remain scarce. The possible reasons are as follows [8, 21, 55]: anticoagulation has serious side effects; PVT does not occur in all patients with cirrhosis; some PVT has a very high rate of spontaneous recanalization; and even if PVT is resolved with the use of anticoagulants, it may recur after stopping treatment. Many scholars have employed preventive anticoagulation after splenectomy or cancer resection in patients with liver cirrhosis. The surgical process and postoperative recovery may lead to a persistent hypercoagulable state, hemodynamic changes of the portal vein system, and local vascular disease, further promoting the occurrence of PVT $[56,57]$. Our data shows that compared with the control group, PVT risk in the observation group does not decrease in patients with liver cirrhosis after cancer resection, but the risk did increase in patients after splenectomy, which is consistent with previous studies $[58,59]$. However, whether preventive anticoagulation should be a routine treatment for patients with liver cirrhosis remains unclear because the study included in this article assessed patients after surgery for cirrhosis. Our data shows that the incidence of bleeding events in the observation group is higher than that in the control group. We believe that preventive anticoagulation is worth considering in those patients at high risk of PVT, such as those undergoing splenectomy.

Given that common anticoagulants have advantages and disadvantages, they should be used with the principle of "individualization." Our results show that the effect of direct oral anticoagulants is improved compared with traditional anticoagulants, and warfarin and aspirin exhibit no significant differences when used in prophylactic anticoagulant therapy. In addition, the combination of traditional Chinese and Western medicine can also achieve good results without increasing the risk of abnormal blood clotting. Intagliata et al. [27] reported that dabigatran or rivaroxaban combined with antiplatelet agents is safer compared with warfarin. Despite these findings, we still need to choose the ideal drug based on the actual situation of the patient. The first factor to consider is pharmacokinetics, especially the functional state of the liver and kidney, which are involved in drug metabolism and clearance. A reduced glomerular filtration rate (GFR) will affect the pharmacokinetics of low molecular 


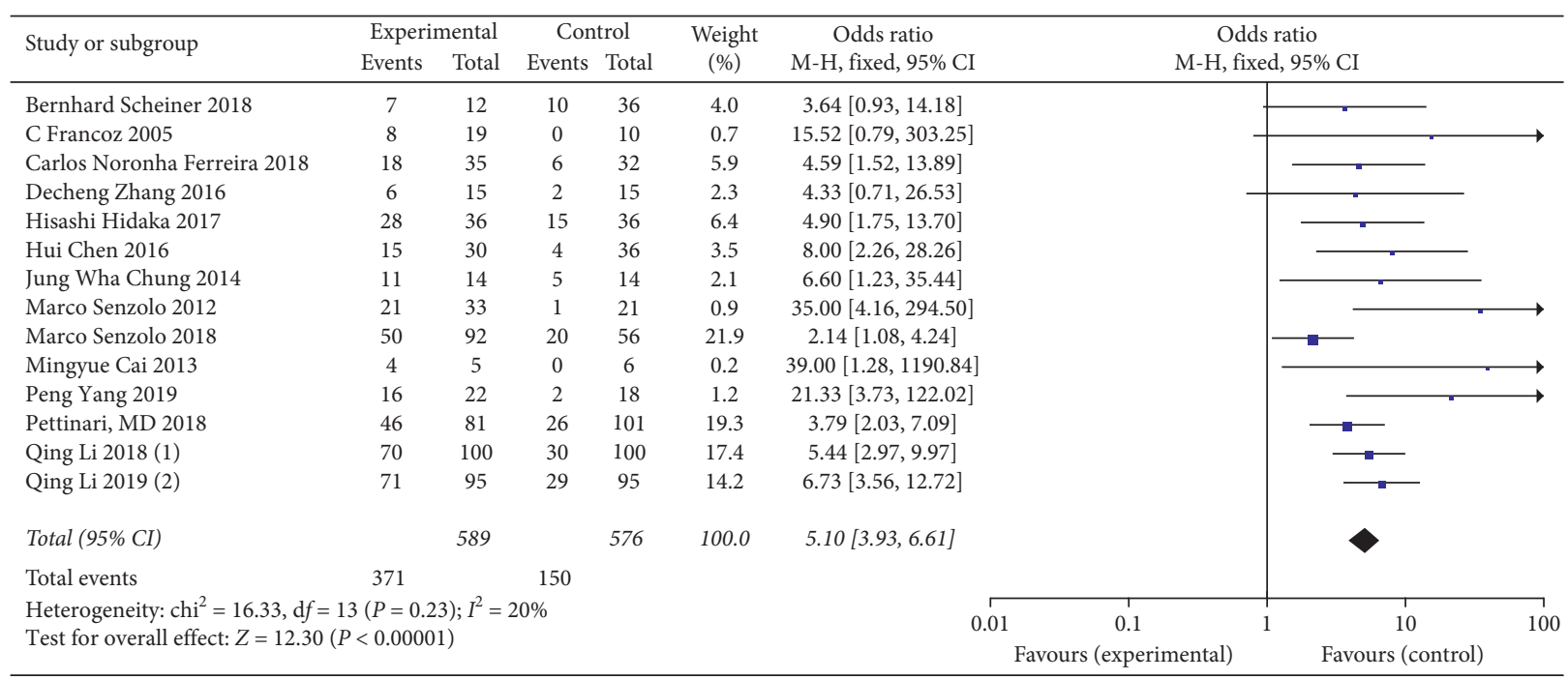

(a)

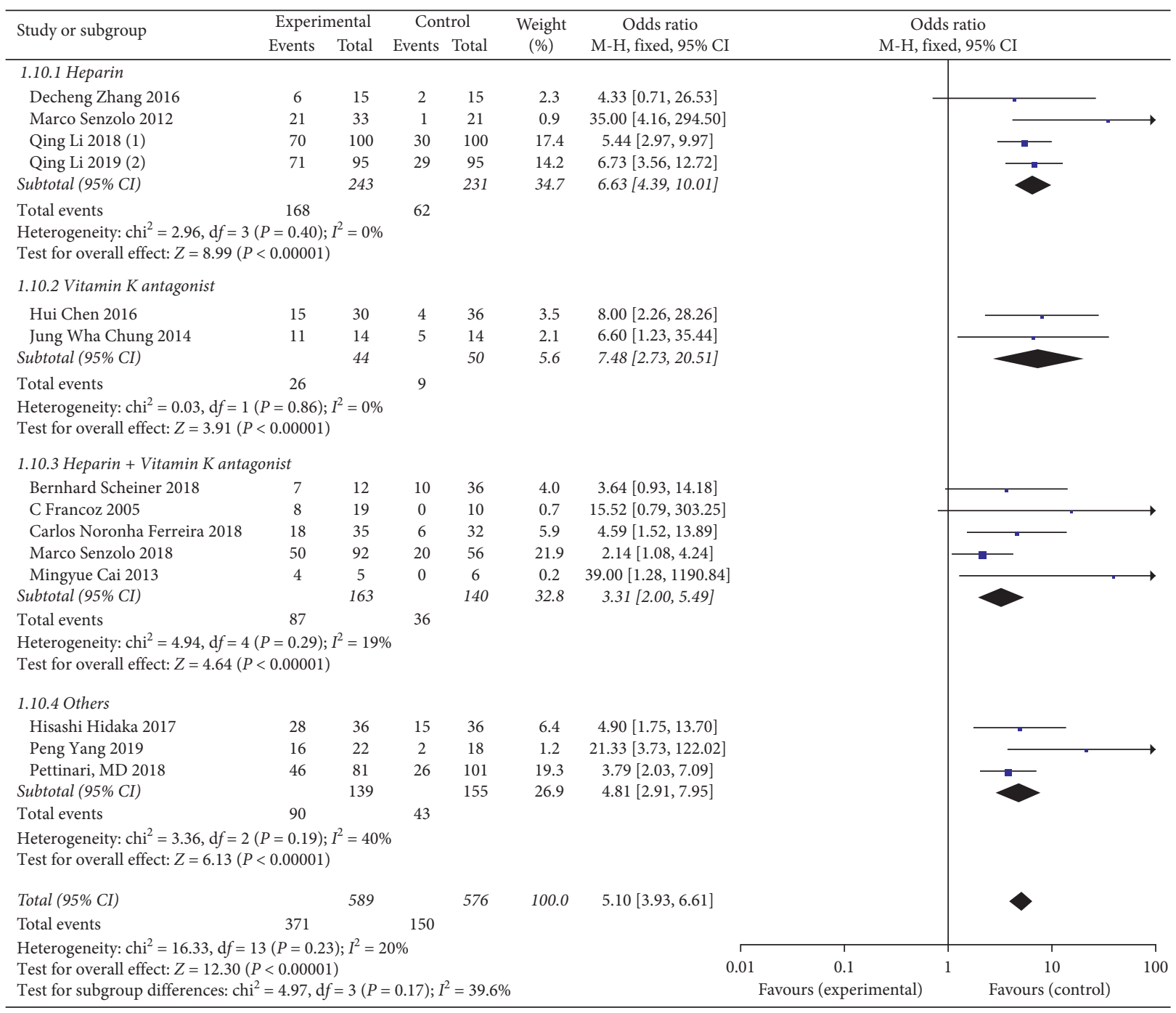

(b)

Figure 3: Continued. 


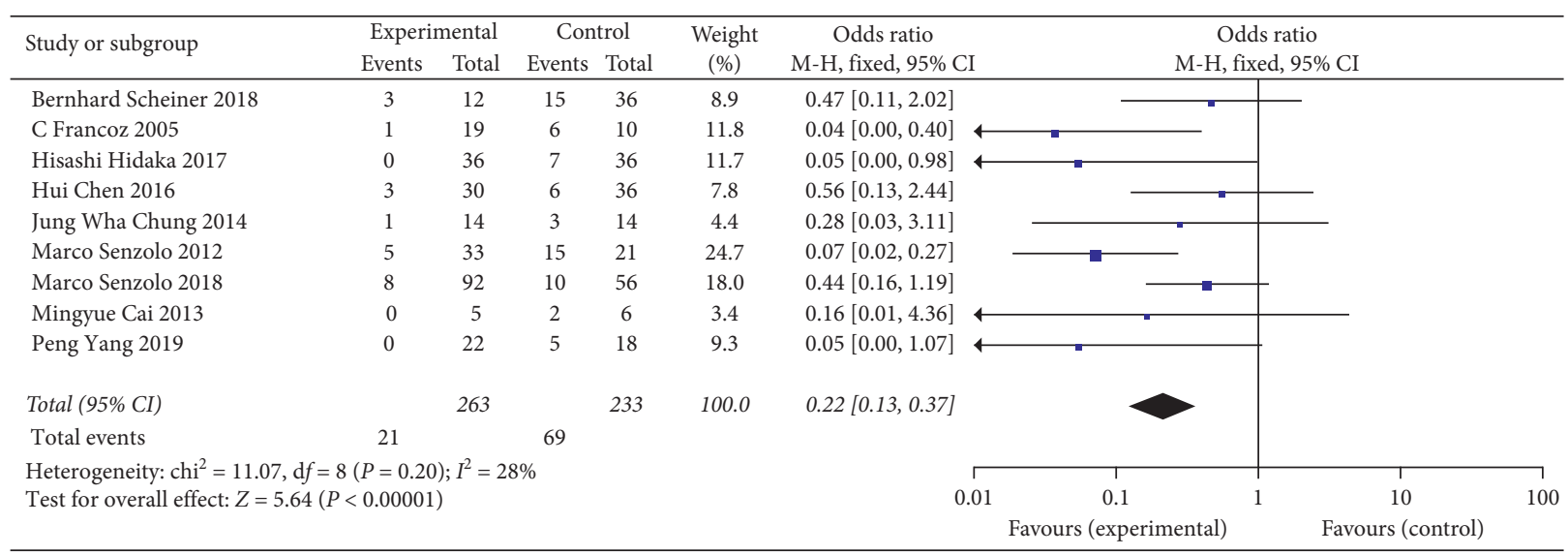

(c)

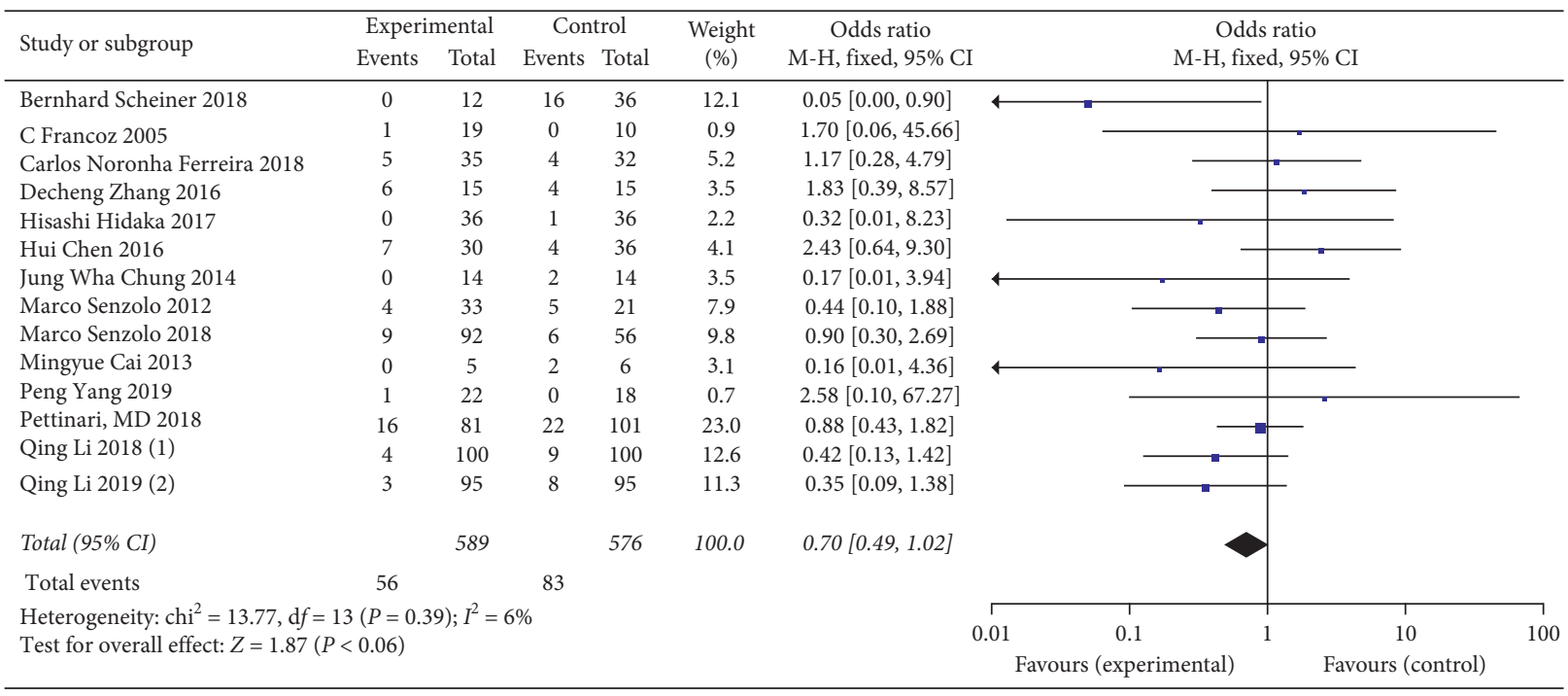

(d)

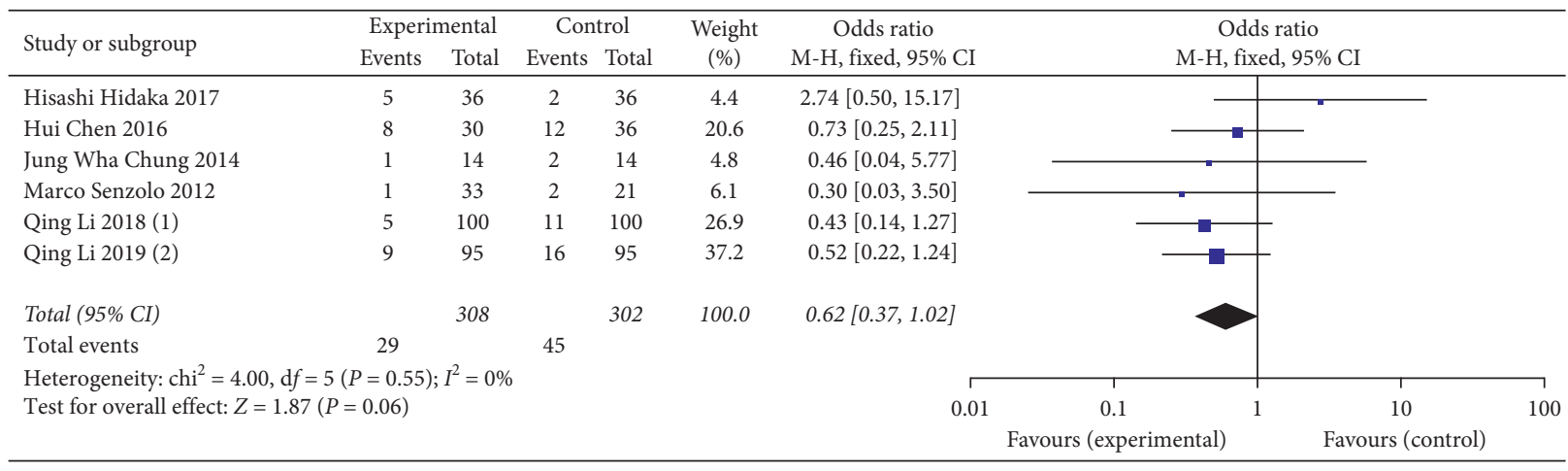

(e)

Figure 3: Continued. 


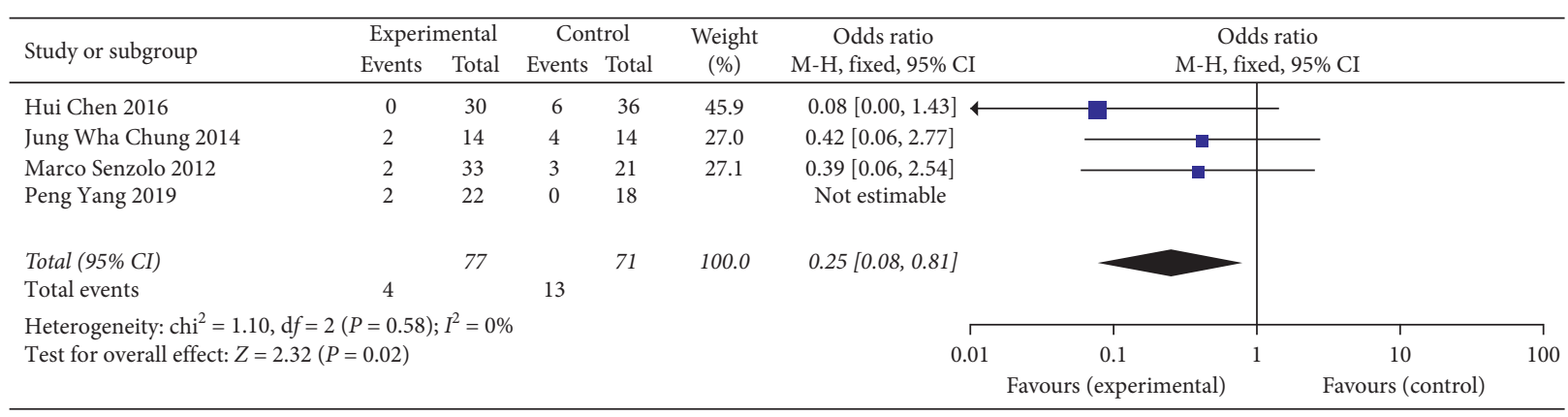

(f)

FIGURE 3: Effect and safety of anticoagulant therapy: (a) analysis of recanalization rate; (b) subgroup analysis of recanalization rate; (c) analysis of thrombus progression or rate of new thrombus formation; (d) bleeding events; (e) other adverse events; (f) mortality rate.

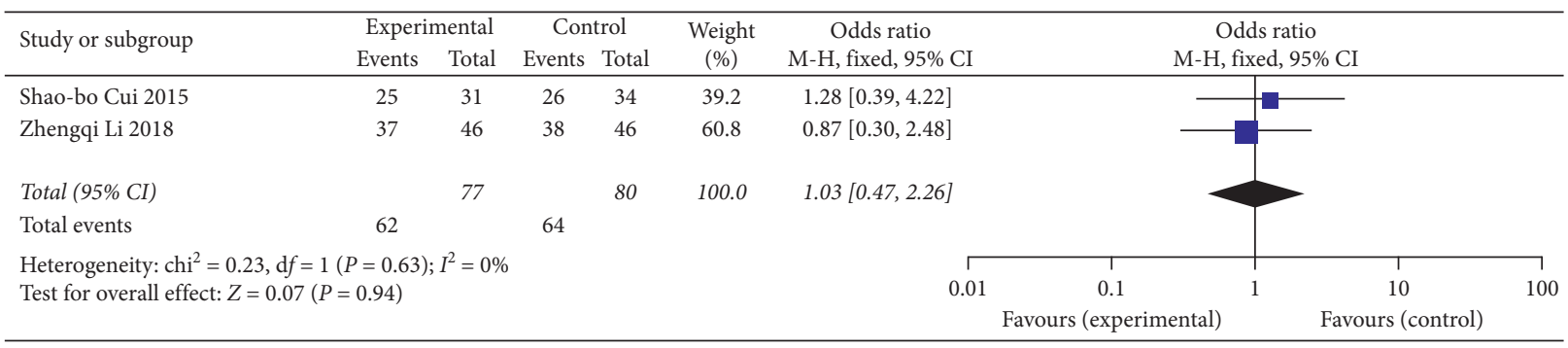

(a)

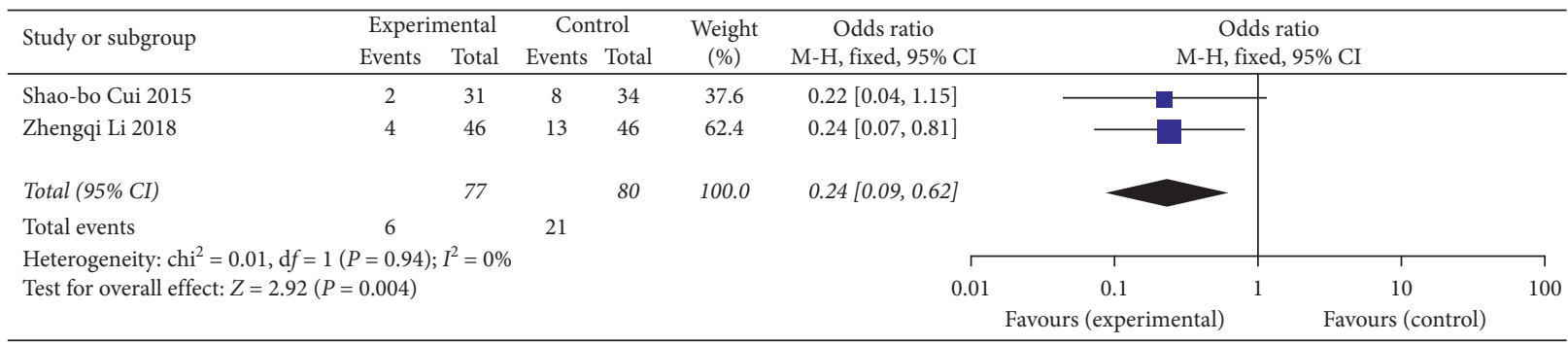

(b)

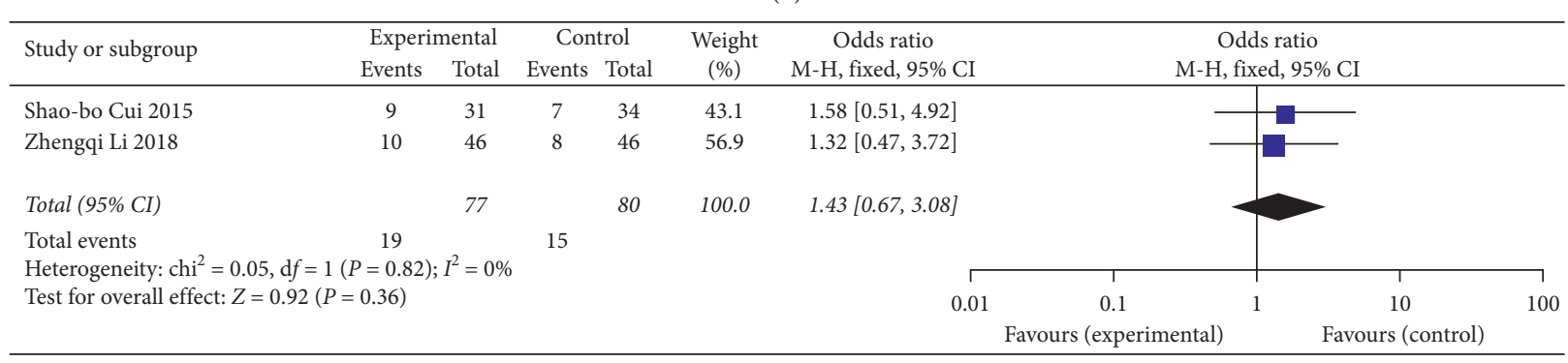

(c)

FIgURe 4: Effect and safety of anticoagulant with different doses of enoxaparin $(1.0 \mathrm{mg} / \mathrm{kg} \mathrm{q} 12 \mathrm{~h}$ in the experimental group and $1.5 \mathrm{mg} / \mathrm{kg}$ qd in the control group): (a) analysis of recanalization rate; (b) bleeding events; (c) other adverse events.

weight heparin (LMWH), and the low density of antithrombin-III in patients with liver cirrhosis may lead to heparin resistance [21]. Patients with renal insufficiency should avoid using dabigatran. The pharmacodynamics of rivaroxaban may be enhanced in patients with liver cirrhosis with poor liver function, while edoxaban, a new oral anticoagulant, is not metabolized by the liver [28, 60]. The interaction of drugs with food and other drugs cannot be ignored. For example, some foods rich in vitamin $\mathrm{K}$ and antibiotics and other drugs can affect the activity of CYP2C9 enzymes and potentially interfere with the efficacy of warfarin [61]. Economic capacity and compliance should also be taken into account. From our results, it seems that heparin is safer than vitamin $\mathrm{K}$ antagonists during the treatment of PVT. However, the high cost, preservation conditions, and daily injection of LMWH cause medical centers to prefer 


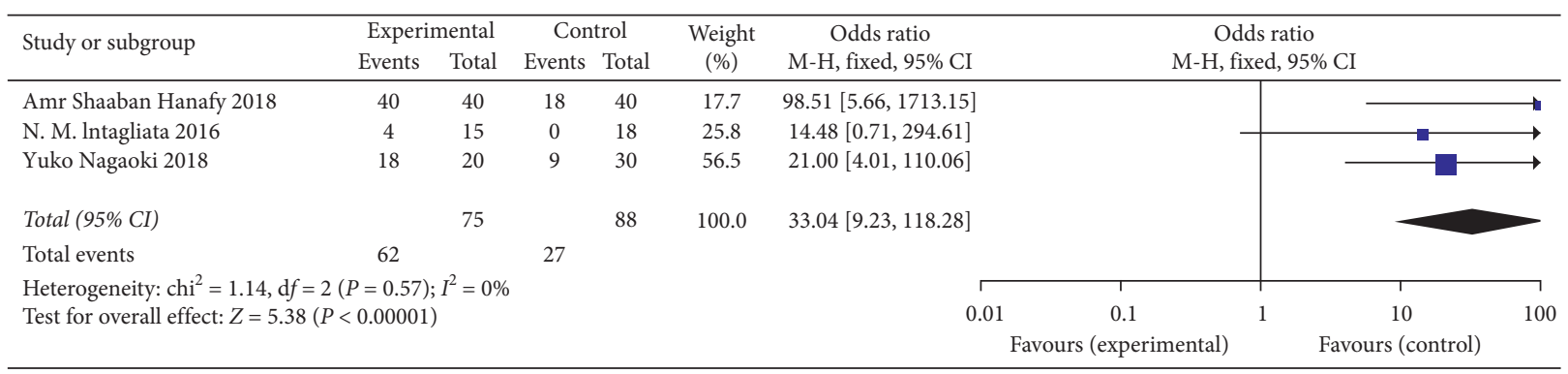

(a)

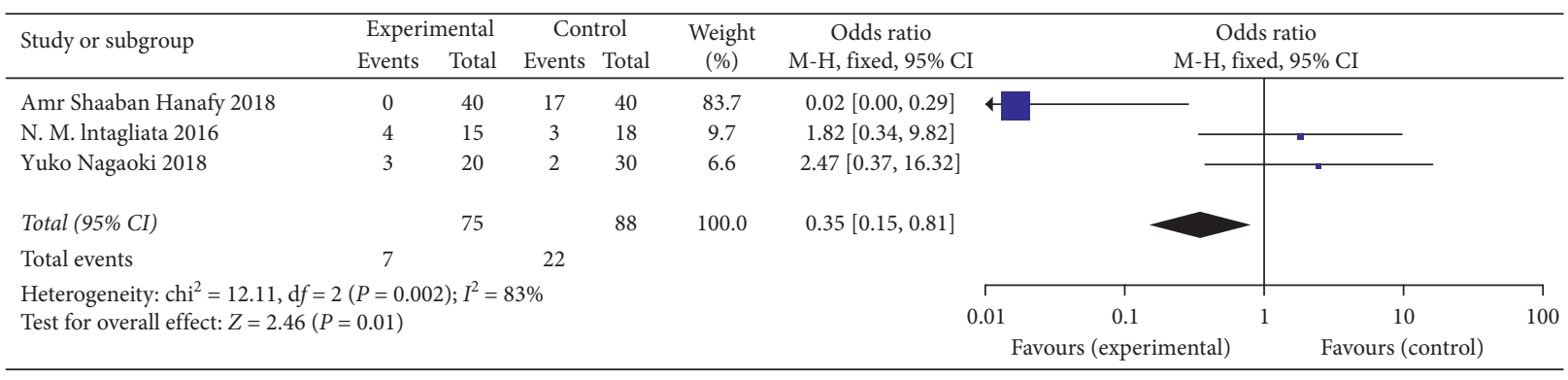

(b)

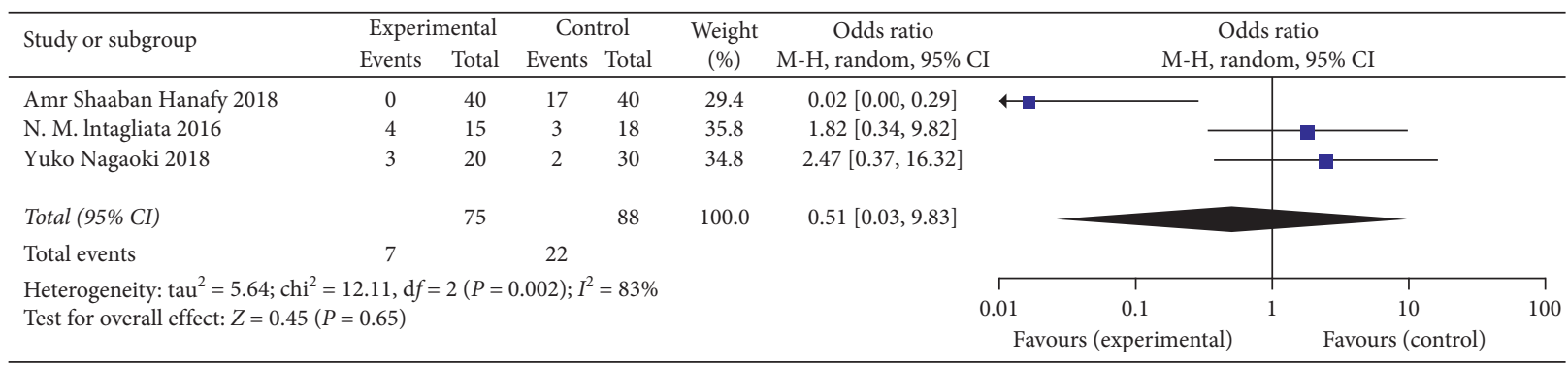

(c)

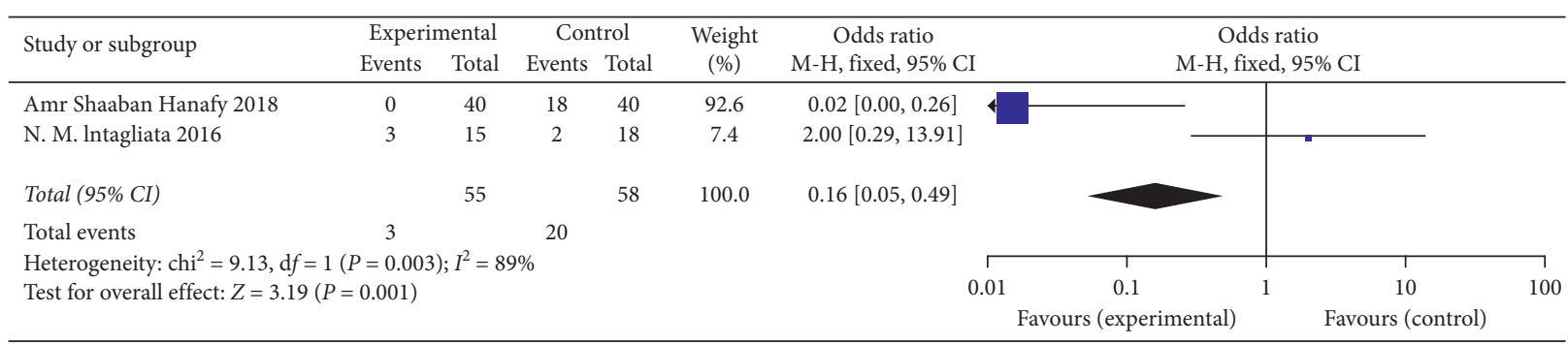

(d)

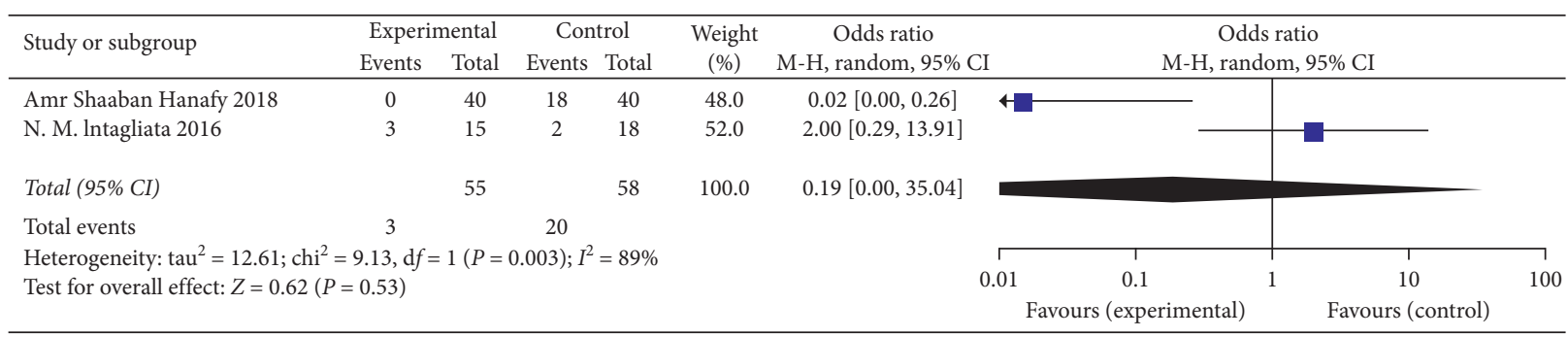

(e)

Figure 5: Continued. 


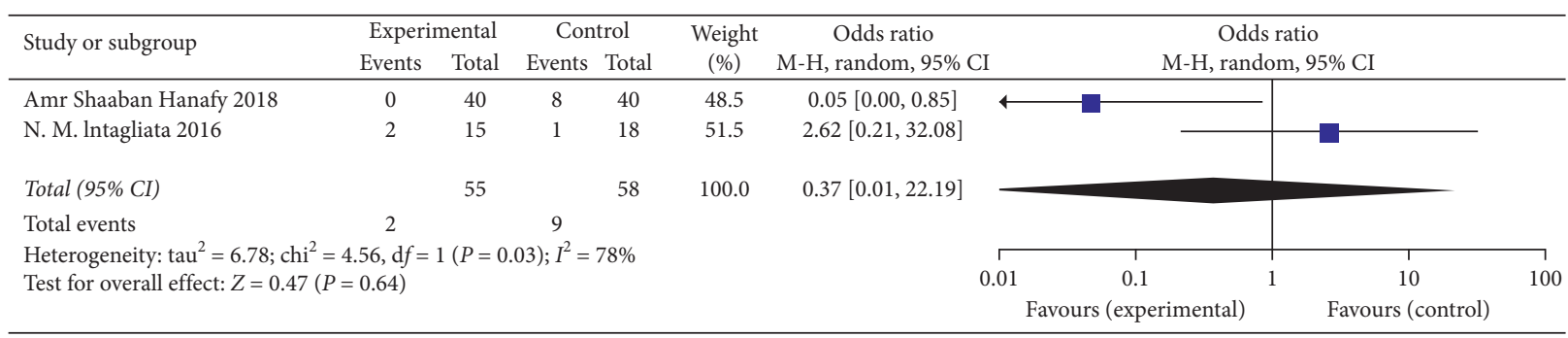

(f)

Figure 5: Therapeutic effects of direct oral anticoagulants vs. traditional anticoagulants (Experimental group: direct oral anticoagulant; Control group: traditional oral anticoagulant): (a) analysis of recanalization rate; (b) bleeding events; (c) analysis of bleeding events after random effects were combined; (d) other adverse events; (e) analysis of other adverse events after random effects were combined; (f) analysis of death events after random effects were combined.

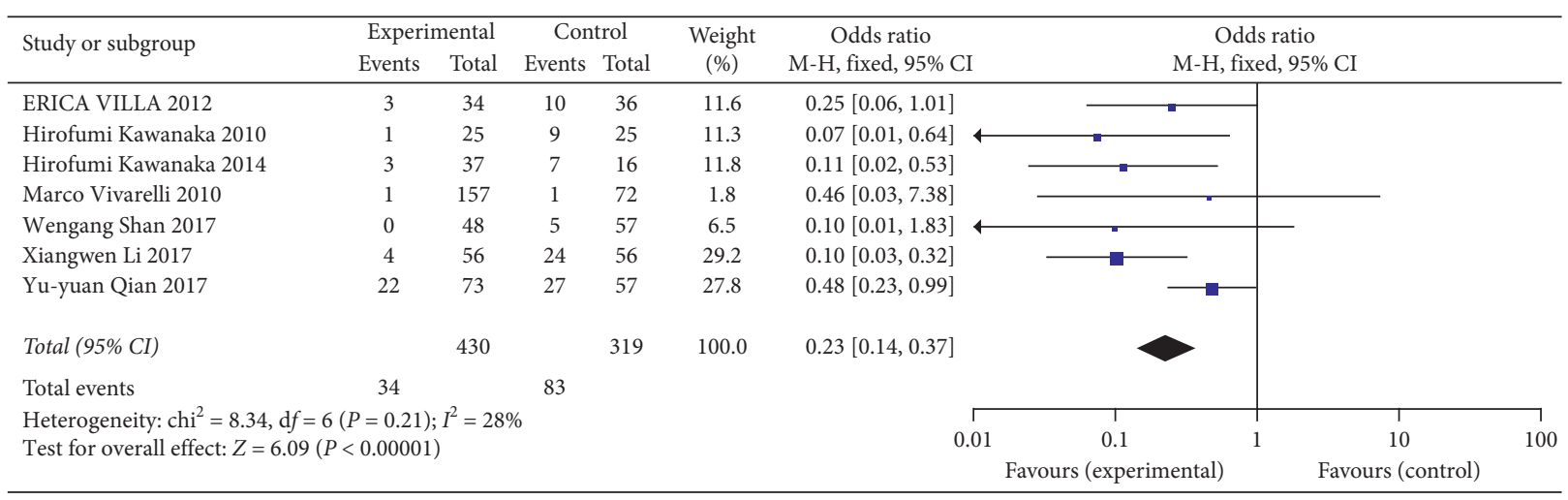

(a)

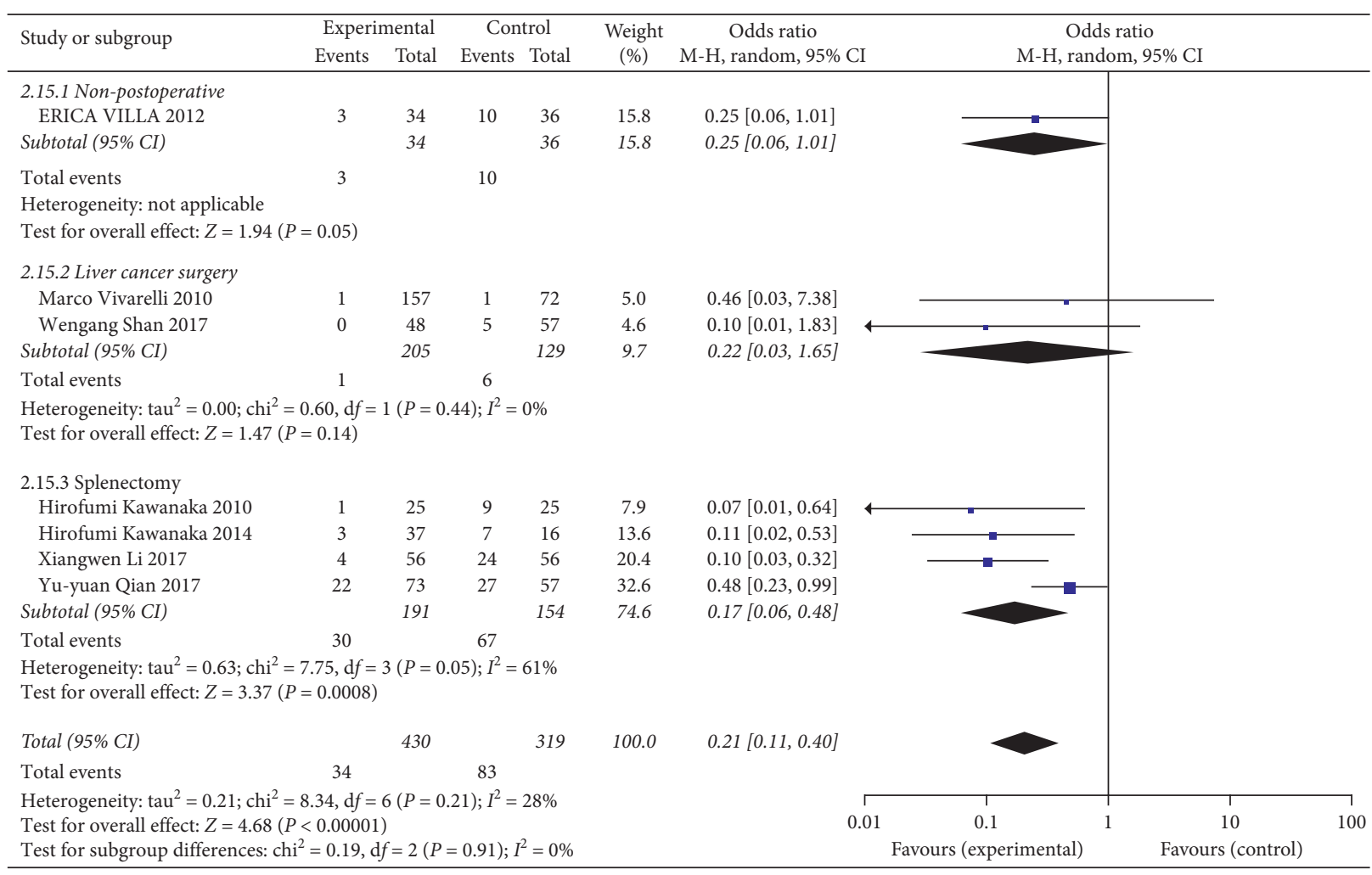

(b)

Figure 6: Continued. 


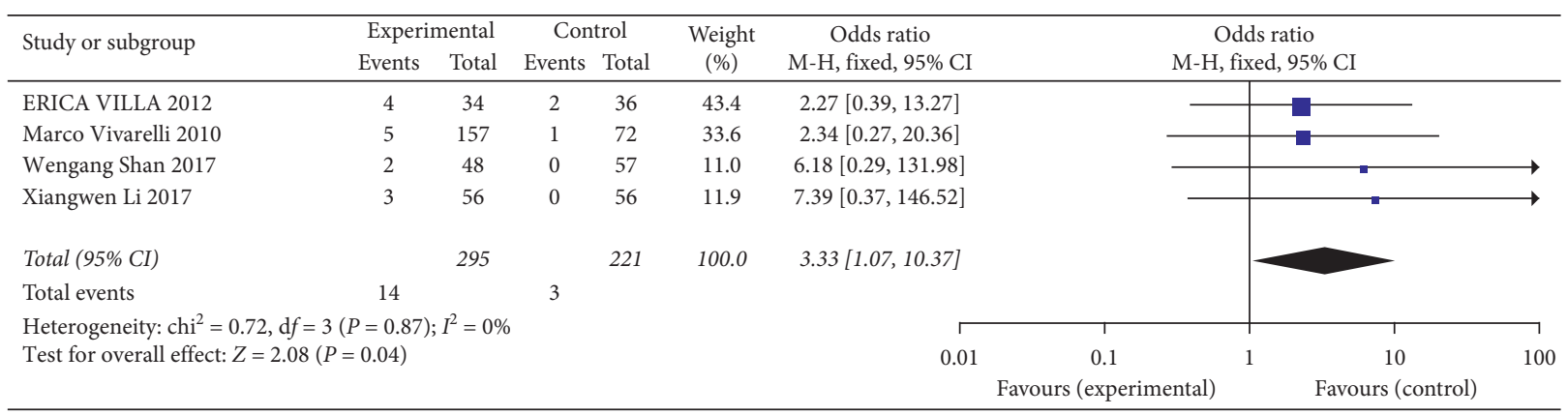

(c)

FiguRE 6: Effect and safety of prophylactic anticoagulant: (a) appearance of new thrombosis; (b) subgroup analysis of new thrombosis; (c) bleeding events.

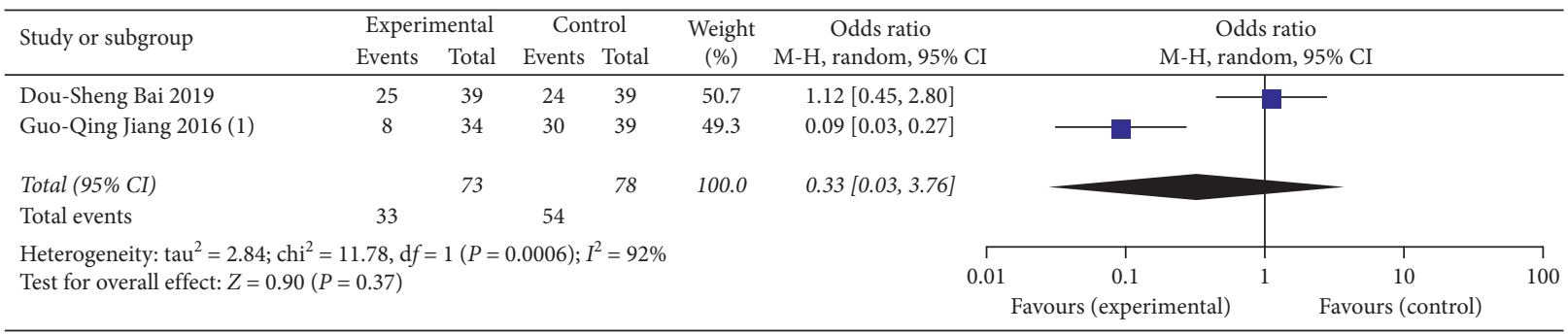

FigURE 7: Effect of prophylactic anticoagulation with different drugs.

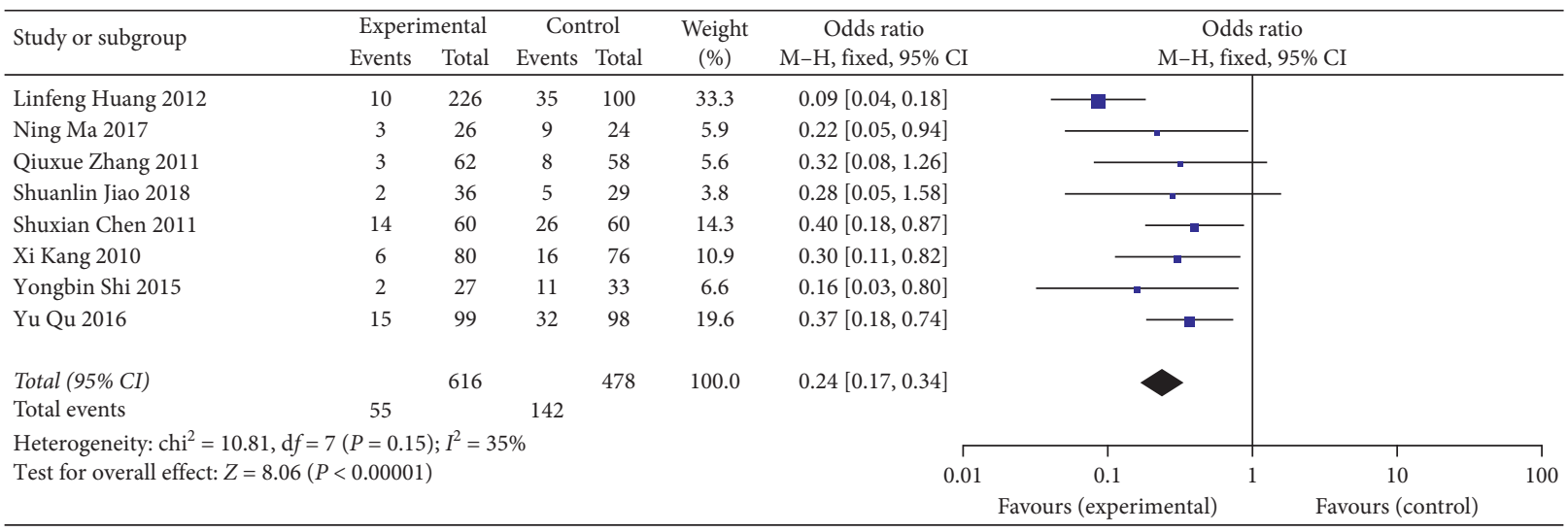

(a)

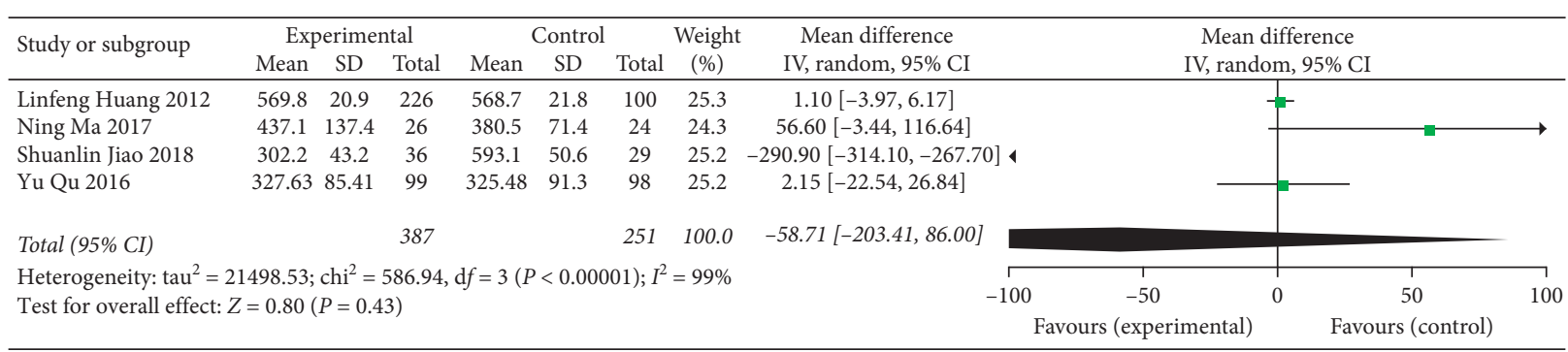

(b)

Figure 8: Continued. 


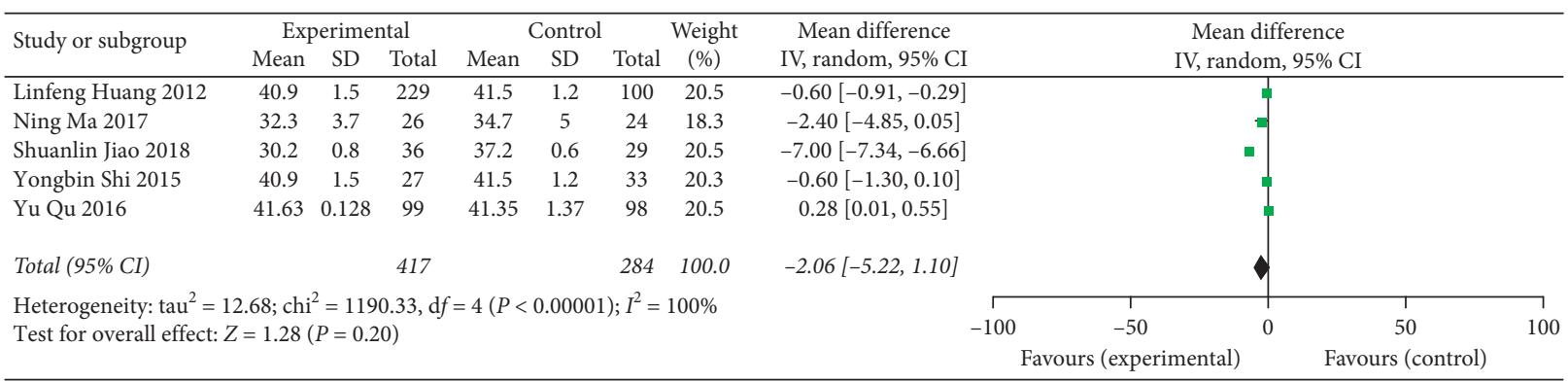

(c)

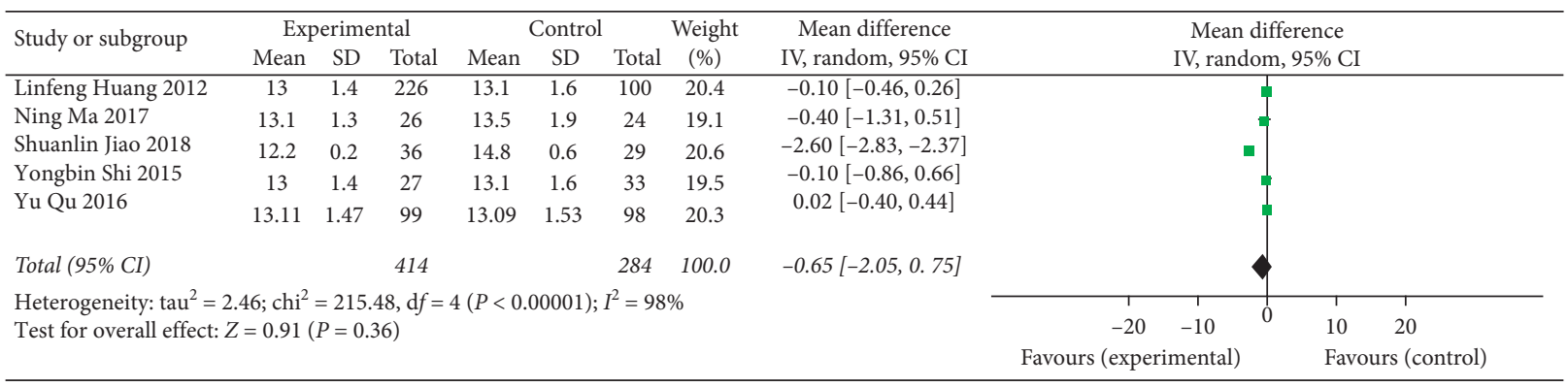

(d)

FIGURE 8: Effect and safety of anticoagulant combined with traditional Chinese medicine: (a) appearance of thrombosis and preventive effect of anticoagulant on PVT; (b) analysis of PLT; (c) analysis of APTT; (d) analysis of PT.

vitamin $\mathrm{K}$ antagonists [62]. For emergency operations, the effect of LMWH exhibits a shorter duration, and the dosage can be adjusted easily and accurately. Thus, LMWH is better than VKA [12]. The Consensus Statement of the 7th Meeting on Coagulation of Liver Disease suggests that it is important to use direct oral anticoagulants (DOACs) as a treatment option for compensatory liver cirrhosis. LMWH is preferred in an emergency, and treatment should continue until hepatic decompensation is stable. In addition, long-term anticoagulation DOACs can be considered as a safe alternative. DOACs are an effective choice for anticoagulant therapy for patients with heparin-induced thrombocytopenia [15].

Next, we should clarify specific treatment dosages and anticoagulant regimens. In the studies included in this article, the dose and timing of anticoagulant drugs are subjective, and currently, no international standard exists for these parameters. Only two articles discussed the use of enoxaparin and found that it is safer to use it in small doses and at multiple times. The anticoagulant time suggested in each guideline or consensus also varies. The American Association for the Study of Liver Diseases (AASLD) recommends anticoagulant therapy for at least 3 months to recanalize the PVT in cases with the deterioration of intestinal infarction and portal hypertension [63]. In 2018, the National Comprehensive Cancer Network (NCCN) recommended anticoagulation for at least 6 months without contraindications [64]. In patients with superior mesenteric vein thrombosis, with a past history suggestive of intestinal ischemia or liver transplant candidates, the European Association for the Study of Liver recommended lifelong anticoagulation [65]. The clinical evidence for these problems is inadequate, and data from more clinical trials are needed to support these findings.

In addition, the effects of anticoagulant therapy are affected by many factors, such as age, liver function score, thrombus condition, platelet count, time of thrombosis, hepatic encephalopathy, and hereditary thrombotic disease $[13,16]$. Delgado et al. [55] proposed that anticoagulant therapy should begin as early as 2 weeks before the discovery of thrombosis because the processes of fibrosis in chronic PVT are irreversible. One study reported that SMV thrombus is an important parameter related to the continuous recanalization of the portal vein. When the PVT extends out of the SMV and the flow rate is reduced by $50 \%$, the anticoagulant effect may be offset by a reduced flow rate [66]. Varicose veins rupture, so bleeding is also associated with PVT recanalization [13]. The 2015 European Guidelines for Hepatic Vascular Disease state that it is important to fully assess the risk of acute bleeding or esophageal and gastric variceal rupture bleeding prior to anticoagulant therapy and to prepare methods to prevent bleeding [65]. It should be noted that approximately $70 \%$ to $75 \%$ of PVTs occur in malignant tumors [67]. The prognosis of patients with tumor thrombus infiltration is extremely poor, so the use of anticoagulants is not recommended. Therefore, attention should also be paid to distinguish a cancer thrombus from a benign thrombus by the combined judgment of imaging features and alpha-fetoprotein levels before anticoagulant treatment [66]. In summary, the clinical decision-making process for anticoagulant therapy requires many comprehensive considerations. 
A major limitation of this study is that some articles are nonrandomized controlled trials. These studies carry a certain level of bias, such as patient selection, drug dosage and course, treatment evaluation, and follow-up. In addition, the lack of patients stratification according to the severity of cirrhosis (compensated/decopensated, CP class A/ $\mathrm{B} / \mathrm{C}, \mathrm{MELD}$, etc....) in the evaluation of treatment effects prevents us from determining whether all patients with cirrhosis should be treated with anticoagulation. Anticoagulant therapy based on combined traditional Chinese and Western medicine seeks to promote blood circulation by preventing blood stasis during PVT treatment. Preventive anticoagulation also requires comparative clinical trials between the anticoagulant with and without traditional Chinese medicine to further confirm the effect on promoting blood circulation and preventing blood stasis. The longest median follow-up time in the study in this paper is 5 years, and the effect of anticoagulants on long-term prognosis requires further study.

\section{Summary}

PVT is a serious adverse event in patients with cirrhosis. The results show that anticoagulant therapy can effectively and safely treat PVT in patients with cirrhosis and effectively reduce the mortality rate. In addition, this paper also demonstrates that prophylactic anticoagulant therapy can prevent PVT after splenectomy. The necessity of prophylactic anticoagulant therapy requires further discussion. In cases without contraindications, anticoagulants are recommended for liver cirrhosis patients with PVT. The selection of anticoagulant drugs and the dosage and course of drugs should be considered based on the patient's conditions.

\section{Data Availability}

The data used to support the findings of this study are included within the article.

\section{Conflicts of Interest}

The authors declare no conflicts of interest.

\section{Authors' Contributions}

Huan Chen and Jiaming Lei contributed equally to this work.

\section{Acknowledgments}

This work was supported by the Special Scientific Research Project of Sichuan Medical Association (Hengrui) about the prevention and treatment of venous embolism (2019HR29), Talent development project of the Affiliated Hospital of Southwest Medical University, and Support Project of Luzhou City Science and Technology Bureau (2016LZXNYD-T06).

\section{Supplementary Materials}

Supplementary Figure 1: histogram of effect and safety of anticoagulant therapy. Supplementary Figure 2: histogram of effect and safety of anticoagulant with different doses of enoxaparin. Supplementary Figure 3: histogram of effect and safety of direct oral anticoagulants vs. traditional anticoagulants. Supplementary Figure 4: histogram of effect and safety of preventive anticoagulant. Supplementary Figure 5: histogram of effect and safety of prophylactic anticoagulation with different drugs. Supplementary Figure 6: histogram of effect and safety of anticoagulant combined with traditional Chinese medicine. (Supplementary Materials)

\section{References}

[1] M.-H. Deng, B. Liu, H. P. Fang et al., "Predictive value of D-dimer for portal vein thrombosis after portal hypertension surgery in hepatitis B virus-related cirrhosis," World Journal of Gastroenterology, vol. 13, no. 48, pp. 6588-6592, 2007.

[2] Y.-y. Qian and K. Li, "The early prevention and treatment of PVST after laparoscopic splenectomy: a prospective cohort study of 130 patients," International Journal of Surgery, vol. 44, pp. 147-151, 2017.

[3] X. Qi, J. Dai, J. Jia et al., "Association between portal vein thrombosis and survival of liver transplant recipients: a systematic review and meta-analysis of observational studies," Journal of Gastrointestinal and Liver Diseases, vol. 24, no. 1, pp. 51-59, 2015.

[4] E. Weinberg, J. Palecki, and K. Reddy, "Direct-acting oral anticoagulants (DOACs) in cirrhosis and cirrhosis-associated portal vein thrombosis," Seminars in Liver Disease, vol. 39, no. 2, pp. 195-208, 2019.

[5] N. Kinjo, H. Kawanaka, T. Akahoshi et al., "Portal vein thrombosis in liver cirrhosis," World Journal of Hepatology, vol. 6, no. 2, pp. 64-71, 2014.

[6] D. J. Harding, M. T. P. Perera, F. Chen, S. Olliff, and D. Tripathi, "Portal vein thrombosis in cirrhosis: controversies and latest developments," World Journal of Gastroenterology, vol. 21, no. 22, pp. 6769-6784, 2015.

[7] B. Condat, F. Pessione, M. Helene Denninger, S. Hillaire, and D. Valla, "Recent portal or mesenteric venous thrombosis: increased recognition and frequent recanalization on anticoagulant therapy," Hepatology, vol. 32, no. 3, pp. 466-470, 2000.

[8] L. Amitrano, M. A. Guardascione, A. Menchise et al., "Safety and efficacy of anticoagulation therapy with low molecular weight heparin for portal vein thrombosis in patients with liver cirrhosis," Journal of Clinical Gastroenterology, vol. 44, no. 6, pp. 448-451, 2010.

[9] M. Senzolo, T. Sartori, V. Rossetto et al., "Prospective evaluation of anticoagulation and transjugular intrahepatic portosistemic shunt for the management of portal vein thrombosis in cirrhosis," Liver International, vol. 32, no. 6, pp. 919-927, 2012.

[10] Z. Wang, M.-S. Jiang, H.-L. Zhang et al., "Is post-TIPS anticoagulation therapy necessary in patients with cirrhosis and portal vein thrombosis? A randomized controlled trial," Radiology, vol. 279, no. 3, pp. 943-951, 2016.

[11] B. Scheiner, P. R. Stammet, S. Pokorny et al., "Anticoagulation in non-malignant portal vein thrombosis is safe and improves hepatic function," Wien Klin Wochenschr, vol. 130, no. 13-14, pp. 446-455, 2018. 
[12] C. Francoz, J. Belghiti, V. Vilgrain et al., "Splanchnic vein thrombosis in candidates for liver transplantation: usefulness of screening and anticoagulation," Gut, vol. 54, no. 5, pp. 691-697, 2005.

[13] C. Noronha Ferreira, D. Reis, H. Cortez-Pinto et al., "Anticoagulation in cirrhosis and portal vein thrombosis is safe and improves prognosis in advanced cirrhosis," Digestive Diseases and Sciences, vol. 64, no. 9, pp. 2671-2683, 2019.

[14] D. Zhang, Analysis of the Risk Factors for Portal Vein Thrombosis in Patients with Liver Cirrhosis and the Efficacy of Anticoagulation Therapy, Anhui Medical University, Heifei, China, 2016.

[15] H. Hidaka, S. Kokubu, T. Sato et al., "Antithrombin III for portal vein thrombosis in patients with liver disease: a randomized, double-blind, controlled trial," Hepatology Research, vol. 48, no. 3, pp. E107-E116, 2018.

[16] H. Chen, L. Liu, X. Qi et al., "Efficacy and safety of anticoagulation in more advanced portal vein thrombosis in patients with liver cirrhosis," European Journal of Gastroenterology \& Hepatology, vol. 28, no. 1, pp. 82-89, 2016.

[17] J. W. Chung, G. H. Kim, J. H. Lee et al., "Safety, efficacy, and response predictors of anticoagulation for the treatment of nonmalignant portal-vein thrombosis in patients with cirrhosis: a propensity score matching analysis," Clinical and Molecular Hepatology, vol. 20, no. 4, pp. 384-391, 2014.

[18] M. Senzolo, N. Riva, F. Dentali et al., "Long-term outcome of splanchnic vein thrombosis in cirrhosis," Clinical and Translational Gastroenterology, vol. 9, no. 8, p. 176, 2018.

[19] M. Cai, K. Zhu, W. Huang et al., "Portal vein thrombosis after partial splenic embolization in liver cirrhosis: efficacy of anticoagulation and long-term follow-up," Journal of Vascular and Interventional Radiology, vol. 24, no. 12, pp. 1808-1816, 2013.

[20] P. Yang, Risk Factors of Portal Vein Thrombosis in Cirrhosis and Efficacy Analysis of Anticoagulant Therapy, Shanxi Medical University, Taiyuan, China, 2019.

[21] I. Pettinari, R. Vukotic, H. Stefanescu et al., "Clinical impact and safety of anticoagulants for portal vein thrombosis in cirrhosis," The American Journal of Gastroenterology, vol. 114, no. 2, pp. 258-266, 2019.

[22] Q. Li, "Effects of low molecular weight heparin anticoagulation in cirrhotic patients with portal vein thrombosis," China Medical Herald, vol. 15, no. 4, pp. 69-72, 2018.

[23] Q. Li, "Analysis of clinical value of low molecular weight heparin sodium in patients with liver cirrhosis and portal vein thrombosis," Modern Digestion \& Intervention, vol. 24, no. 10, pp. 1137-1139, 2019.

[24] S.-b. Cui, R.-h. Shu, S.-p. Yan et al., "Efficacy and safety of anticoagulation therapy with different doses of enoxaparin for portal vein thrombosis in cirrhotic patients with hepatitis B," European Journal of Gastroenterology \& Hepatology, vol. 27, no. 8, pp. 914-919, 2015.

[25] Z. Li, "Dose selection. efficacy and risk analysis of early enoxaparin anticoagulation in the treatment of liver cirrhosis complicated with portal vein thrombosis," Chinese Journal of Integrated Traditional and Western Medicine on Liver Disease, vol. 28, no. 4, pp. 151-153, 2018.

[26] A. S. Hanafy, S. Abd-Elsalam, and M. M. Dawoud, "Randomized controlled trial of rivaroxaban versus warfarin in the management of acute non-neoplastic portal vein thrombosis," Vascular Pharmacology, vol. 113, pp. 86-91, 2019.

[27] N. M. Intagliata, Z. H. Henry, H. Maitland et al., "Direct oral anticoagulants in cirrhosis patients pose similar risks of bleeding when compared to traditional anticoagulation,"
Digestive Diseases and Sciences, vol. 61, no. 6, pp. 1721-1727, 2016.

[28] Y. Nagaoki, H. Aikata, K. Daijyo et al., "Efficacy and safety of edoxaban for treatment of portal vein thrombosis following danaparoid sodium in patients with liver cirrhosis," Нepatology Research, vol. 48, no. 1, pp. 51-58, 2018.

[29] E. Villa, C. Cammà, M. Marietta et al., "Enoxaparin prevents portal vein thrombosis and liver decompensation in patients with advanced cirrhosis," Gastroenterology, vol. 143, no. 5, pp. 1253-1260, 2012.

[30] H. Kawanaka, T. Akahoshi, N. Kinjo et al., "Impact of antithrombin III concentrates on portal vein thrombosis after splenectomy in patients with liver cirrhosis and hypersplenism," Annals of Surgery, vol. 251, no. 1, pp. 76-83, 2010.

[31] H. Kawanaka, T. Akahoshi, S. Itoh et al., "Optimizing risk stratification in portal vein thrombosis after splenectomy and its primary prophylaxis with antithrombin III concentrates and danaparoid sodium in liver cirrhosis with portal hypertension," Journal of the American College of Surgeons, vol. 219, no. 5, pp. 865-874, 2014.

[32] M. Vivarelli, M. Zanello, C. Zanfi et al., "Prophylaxis for venous thromboembolism after resection of hepatocellular carcinoma on cirrhosis: is it necessary?" World Journal of Gastroenterology, vol. 16, no. 17, pp. 2146-2150, 2010.

[33] W. Shan, J. Rao, X. Cheng et al., "The clinical preventive effects of low molecular weight heparin as prophylaxis against venous thromboembolism after primary hepatocellular carcinoma surgery," Acta Universitatis Medicinalis Nanjing, vol. 37, no. 5, pp. 593-596, 2017.

[34] X. Li and M. Tu, "Clinical study of low molecular weight heparin in early prevention of portal vien thrombosis in patients with liver cirrhosis after laparoscopic splenectomy with esophagogatric devascularization," Journal of Practical Hepatology, vol. 20, no. 2, 2017.

[35] D.-S. Bai, B.-L. Xia, C. Zhang et al., "Warfarin versus aspirin prevents portal vein thrombosis after laparoscopic splenectomy and azygoportal disconnection: a randomized clinical trial," International Journal of Surgery, vol. 64, pp. 16-23, 2019.

[36] G.-Q. Jiang, B.-L. Xia, P. Chen et al., “Anticoagulation therapy with warfarin versus low-dose aspirin prevents portal vein thrombosis after laparoscopic splenectomy and azygoportal disconnection," Journal of Laparoendoscopic \& Advanced Surgical Techniques, vol. 26, no. 7, pp. 517-523, 2016.

[37] L. Huang, L. Fan, Y. Qu et al., "Influence of activating bood and anticoagulation therapy in the earlier period on the formation of portal vein thrombosis after devasculariztion," China Medical Herald, vol. 11, no. 13, pp. 33-38, 2014.

[38] M. Ning, Analysis of Portal Vein Thrombosis of Liver Cirrhosis Portal Hypertension after Surgery by Prophylactic Anticoagulation, Jilin University, Changchun, China, 2017.

[39] Q. Zhang, T. Wang, and D. Yang, "Observation of the curative effect of anticoagulants on preventing portal vein thrombosis after splenectomy in patients with cirrhosis and hypersplenism," Hebei Medicine, vol. 33, no. 9, pp. 1358-1359, 2011.

[40] S. Jiao, X. Zhao, S. Du et al., "High-dose compound salvia miltiorrhiza combined with low-dose low molecular heparin to prevent portal vein thrombosis after splenectomy in patients with liver cirrhosis," Chinese Hepatology, vol. 23, no. 7, pp. 623-625, 2018.

[41] S. Chen, J. Lin, Y. Ling et al., "Precautionary effect on the early application of anticoagulant drugs to portal vein thrombosis in patients with portal hypertension after operation," China Medical Herald, vol. 8, no. 29, pp. 73-74, 2011. 
[42] X. Kang and Q. Zhang, "Early application of low molecular weight heparin and salvia miltiorrhiza to prevent portal vein thrombosis after splenectomy and dehydration in patients with liver cirrhosis," Clinical Medicine Practice, vol. 19, no. 1, pp. 16-33, 2010.

[43] Y. Shi, G. Yang, and H. Huang, "Promoting blood circulation and anticoagulant drugs to prevent portal vein thrombosis after splenectomy in patients with portal hypertension," Chinese Journal of Integrative Medicine on Cardio/Cerebrovascular Disease, vol. 13, no. 16, pp. 1911-1912, 2015.

[44] Y. Qu, "Effect of traditional Chinese medicine and western medicine in the prevention of portal vein thrombosis after surgery," International Journal of Geriatrics, vol. 37, no. 2, pp. 69-72, 2016.

[45] A. Luca, S. Caruso, M. Milazzo et al., "Natural course of extrahepatic nonmalignant partial portal vein thrombosis in patients with cirrhosis," Radiology, vol. 265, no. 1, pp. 124-132, 2012.

[46] B. V. John, R. Konjeti, A. Aggarwal et al., "Impact of untreated portal vein thrombosis on pre and post liver transplant outcomes in cirrhosis," Annals of Hepatology, vol. 12, no. 6, pp. 952-958, 2013.

[47] J. Kwon, Y. Koh, S. j. Yu, and J.-H. Yoon, "Low-molecularweight heparin treatment for portal vein thrombosis in liver cirrhosis: efficacy and the risk of hemorrhagic complications," Thrombosis Research, vol. 163, pp. 71-76, 2018.

[48] G. Silva, F. Turon, V. Hernandez-Gea et al., "Unexpected disappearance of portal cavernoma on long-term anticoagulation," Journal of Hepatology, vol. 61, no. 2, pp. 446448, 2014.

[49] M. J. Garcia-Fuster, N. Abdilla, M. J. Fabia et al., "Venous thromboembolism and liver cirrhosis," Revista Espanola De Enfermedades Digestivas, vol. 100, no. 5, pp. 259-262, 2008.

[50] P. Northup and B. Reutemann, "Management of coagulation and anticoagulation in liver transplantation candidates," Liver Transplantation, vol. 24, no. 8, pp. 1119-1132, 2018.

[51] F. Cerini, M. Vilaseca, E. Lafoz et al., "Enoxaparin reduces hepatic vascular resistance and portal pressure in cirrhotic rats," Journal of Hepatology, vol. 64, no. 4, pp. 834-842, 2016.

[52] K. Brusilovskaya, P. Königshofer, P. Schwabl, and T. Reiberger, "Vascular targets for the treatment of portal hypertension," Seminars in Liver Disease, vol. 39, no. 4, pp. 483-501, 2019.

[53] N. M. Intagliata, C. K. Argo, J. G. Stine, T. Lisman, S. H. Caldwell, and F. Violi, "Concepts and controversies in haemostasis and thrombosis associated with liver disease: proceedings of the 7 th international coagulation in liver disease conference," Thrombosis and Haemostasis, vol. 118, no. 08, pp. 1491-1506, 2018.

[54] Y. Peng, x. Qi, and x. Guo, "Recommendations for consensus statement by the Italian Association for the study of liver diseases (AISF) and the Italian society of Internal medicine (SIMI): hemostantic balance in patients with liver cirrhosis," J Clin Hepatol, vol. 32, no. 6, pp. 1052-1053, 2016.

[55] M. G. Delgado, S. Seijo, I. Yepes et al., "Efficacy and safety of anticoagulation on patients with cirrhosis and portal vein thrombosis," Clinical Gastroenterology and Hepatology, vol. 10, no. 7, pp. 776-783, 2012.

[56] J. Salinas, D. Barros, N. Salgado et al., "Portomesenteric vein thrombosis after laparoscopic sleeve gastrectomy," Surgical Endoscopy, vol. 28, no. 4, pp. 1083-1089, 2014.

[57] A. Tripodi and P. M. Mannucci, "The coagulopathy of chronic liver disease," New England Journal of Medicine, vol. 365, no. 2, pp. 147-156, 2011.
[58] W. Zhang, D. M. Zhou, and Y. Li, "Clinical effect of lowmolecular-weight heparin in prevention and treatment of liver cirrhosis and portal vein thrombosis after splenectomy: a systematic review and meta-analysis]," Zhonghua Gan Zang Bing Za Zhi, vol. 24, no. 10, pp. 732-737, 2016.

[59] X. Zhang, Y. Wang, M. Yu, J. Huang, D. Deng, and H. Xue, "Effective prevention for portal venous system thrombosis after splenectomy: a meta-analysis," Journal of Laparoendoscopic \& Advanced Surgical Techniques, vol. 27, no. 3, pp. 247-252, 2017.

[60] A. G. Turpie, R. Kreutz, J. Llau, B. Norrving, and S. Haas, "Management consensus guidance for the use of rivaroxaban--an oral, direct factor Xa inhibitor," Thrombosis and Haemostasis, vol. 108, no. 5, pp. 876-886, 2012.

[61] D. R. Flora, A. E. Rettie, R. C. Brundage, and T. S. Tracy, "CYP2C9 genotype-dependent warfarin pharmacokinetics: impact of CYP2C9 genotype on R- and S-warfarin and their oxidative metabolites," The Journal of Clinical Pharmacology, vol. 57, no. 3, pp. 382-393, 2017.

[62] E. G. Giannini and V. Savarino, "Which anticoagulant drug should be used to treat portal vein thrombosis in patients with chronic liver disease?" Clinical Gastroenterology and Hepatology, vol. 11, no. 1, p. 103, 2013.

[63] L. D. DeLeve, D.-C. Valla, and G. Garcia-Tsao, "Vascular disorders of the liver," Hepatology, vol. 49, no. 5, pp. 1729-1764, 2009.

[64] M. B. Streiff, B. Holmstrom, D. Angelini et al., "NCCN guidelines insights: cancer-associated venous thromboembolic disease, version 2.2018," Journal of the National Comprehensive Cancer Network, vol. 16, no. 11, pp. 1289-1303, 2018.

[65] J. C. Garcia-Pagan, E. Buscarini, H. L. A. Janssen et al., "EASL clinical practice guidelines: vascular diseases of the liver," Journal of Hepatology, vol. 64, no. 1, pp. 179-202, 2016.

[66] N. M. Intagliata, S. H. Caldwell, and A. Tripodi, "Diagnosis, development, and treatment of portal vein thrombosis in patients with and without cirrhosis," Gastroenterology, vol. 156, no. 6, pp. 1582-1599, 2019.

[67] M. Wu, M. Schuster, and M. Tadros, "Update on management of portal vein thrombosis and the role of novel anticoagulants," Journal of Clinical and Translational Hepatology, vol. 7, no. 2, pp. 154-164, 2019. 